\title{
DERIVED CATEGORIES AND THE ANALYTIC APPROACH TO GENERAL RECIPROCITY LAWS. PART I
}

MICHAEL BERG

Received 21 December 2004

We reformulate Hecke's open problem of 1923, regarding the Fourier-analytic proof of higher reciprocity laws, as a theorem about morphisms involving stratified topological spaces. We achieve this by placing Kubota's formulations of $n$-Hilbert reciprocity in a new topological context, suited to the introduction of derived categories of sheaf complexes. Subsequently, we begin to investigate conditions on associated sheaves and a derived category of sheaf complexes specifically designed for an attack on Hecke's eighty-year-old challenge.

\section{Introduction}

Around 1923, Hecke gave what is often called the analytic proof of quadratic reciprocity for an arbitrary number field, including it as the showpiece of his famous work [15]. Hecke's proof is Fourier analytic, given that there resides at its core the derivation of a functional equation for a (Hecke-) $\vartheta$-function by classical Fourier-analytic means. Evidently, this tactic is based on a classical method of Cauchy [15, page 218]. Some forty years after Hecke's work, this theme of quadratic reciprocity via Fourier analysis was taken up by Weil in his seminal paper [26], where the matter was fitted into the greater context of Siegel's analytic theory of quadratic forms. Weil demonstrated that Hecke's Fourieranalytic maneuvers are equivalent to the fact that the double cover of the adelization of the symplectic group for a number field is split on the rational points. Tellingly, this splitting is due to the invariance of the so-called Weil $\Theta$-functional under the natural action of the rational points as facilitated by the projective Weil representation. At a deeper level all this is part and parcel of Hecke's 9 -functional equations. To boot, Weil's derivation of the indicated critical invariance ultimately comes about by means of a generalization of nothing less than Poisson summation. But something of a paradigm shift has occurred, from $\vartheta$-functions and their functional equations (classical Fourier analysis), to unitary representation theory and low-degree cohomology of local as well as adelic algebraic groups (abstract Fourier analysis). After all, the aforementioned double symplectic cover is essentially uniquely determined by a suitable 2-cocycle. 
Maybe it is not inappropriate, in light of the foregoing, to suggest that a proper broader context for this approach to quadratic reciprocity is that of correspondences and duality theories. Indeed, Hecke's use of $\vartheta$-functional equations points back to Riemann's second proof of the functional equation for his famous $\zeta$-function $[8,23]$ which, in turn, can be regarded as a prototype for the Hecke correspondence(s) [13, 14, 27]. Also, in [26], Weil, at the pivotal stage of proving the invariance property of his $\Theta$-functional, employs a transfer-of-structure tactic essentially through maneuvers with a Fourier transform. Finally, in [3] we took up the related theme of the interplay between Weil indices (which are closely connected to the local 2-cocycles determining local double symplectic covers and coming from the projective Weil representation) and local constants, that is, local Artin root numbers as given in Tate's thesis [24] (and attached to $L$-functions). We propose, as an ideological motivation for what follows in this paper, the thesis that the proper next step is revealed by Grothendieck and his school: functional equations for special functions should be allowed to evolve into, for example, Verdier duality [7], and the fundamental tool of the Fourier transform should be cast as an avatar due to Deligne, Mukai, or Sato (see, e.g., $[5,6,16,22]$ ). But we are getting ahead of ourselves.

Doubtless, a little belatedly, then, we ask the obvious question of why we should concern ourselves with this hackneyed theme of quadratic reciprocity in the first place. The answer is that Hecke's punchline in [15] was a challenge to carry out for general reciprocity laws (of arbitrary degree) what he had just done for quadratic reciprocity. Hecke asked for counterparts to his $\vartheta$-functions, capable of filling a similar role to the latter but for higher reciprocity laws. In [2], we addressed this question directly, that is, purposely naively, and proved that a natural generalization of Hecke's $\vartheta$-functions, built on a form of higher (even) degree in place of the fundamental quadratic form defining a $\vartheta$-function, satisfies a functional equation of altogether forbidding complexity. This circumstance dovetails, as it were, with the algebraic philosophy behind Weil's reformulation of the matter in unitary group representation-theoretic terms. To be precise, the specific question of generalization was left aside by Weil in [26] but was taken up a little later, in 1967 and 1969, by Kubota, in [19, 20]. With Kubota the focus falls specifically on splitting properties (relative to the respective sets of rational points) of metaplectic groups, this being Weil's term for the indicated generalizations of the symplectic group with the double cover discussed above being the most fundamental one. Kubota succeeded in showing that $n$-Hilbert reciprocity is equivalent to having the $n$-fold (metaplectic) cover of the adelization of $\mathrm{SL}_{2}$ over the given global number field split on the respective rational points. Unfortunately, no independent proof of this splitting (without presupposing reciprocity) was available and so Hecke's challenge went unmet due to circularity. This is substantially the present state of affairs.

Granting, then, that (i) the methodology of the Fourier-analytic approach to quadratic reciprocity evolves into something like Grothendieck's approaches to duality and that (ii) Hecke's eighty-year-old challenge can be met by showing that a certain metaplectic group is split on its rational points, we propose in this article to develop Kubota's formalism in the ("dual") setting of the structure sheaves attached to certain topological spaces, namely, the spaces naturally associated to algebraic groups which conspire to realize the critical metaplectic group in a short exact sequence. What emerges 
is that the all-important splitting behavior manifests itself as a four-part proposition (see Proposition 5.1 below) about a commutative diagram in the category of topological spaces. This proposition sets the stage for the tactic of applying methods from the subject of derived categories and perhaps even perverse sheaves.

The structure of the present paper is as follows. Section 2 is an account of the analytic proof of 2-Hilbert reciprocity based on Weil's work in [26], and on Kubota's work in $[19,20]$. The reader is referred to [4] for a more thorough dissection of this material. Next, Section 3 is a presentation of Kubota's generalization of the quadratic formalism to the general case, $n \geq 2$; here, the main reference is [20], of course, but see also Matsumoto [21] and Kazhdan and Patterson [17]. In Section 4, we go into the question of realizing the adelic groups that populate Kubota's short exact sequences in topological terms and address the issue of stratification, anticipating a possible later advent of perverse sheaves. In Section 5, we give the aforementioned formulation of the pivotal splitting property of Kubota's metaplectic group in diagrammatical terms in the category of topological spaces. Section 6 is concerned with the relevant diagrams of topological spaces as sites for sheaves and introduces the dual formulation of Kubota's formalism in terms of sheaves. In Section 7, the machinery of derived categories is introduced, and we begin to close in on our dualized splitting property by means of a theorem about a certain long exact Hom sequence. Finally, in Section 8, we look toward what lies ahead.

\section{Quadratic reciprocity: the double cover of $\mathrm{SL}_{2}$}

Let $k$ be a global algebraic number field with $k^{*}$ its dual space. Then, with $x \in k$ and $y^{*} \in k^{*}$, get a nondegenerate bilinear form in $\left(x, y^{*}\right) \stackrel{B}{\mapsto} y^{*}(x)$; it can be regarded as an element of $H^{2}\left(k \oplus k^{*}, \mathbf{C}_{1}^{\times}\right)$and so defines an extension of $k \oplus k^{*}$ by $\mathbf{C}_{1}^{\times}$whose group law is twisted by $B$. This central extension is Heis $(k)$, the Heisenberg group attached to $k$. With $\mathfrak{p}$ any place of $k$ we get $k \subset k_{\mathfrak{p}}$ and $k^{*} \subset k_{\mathfrak{p}}^{*}$ and can extend $B$ to $B_{\mathfrak{p}}$ on $k_{\mathfrak{p}} \oplus k_{\mathfrak{p}}^{*}$; this results in an element of $H^{2}\left(k_{\mathfrak{p}} \oplus k_{\mathfrak{p}}^{*}, \mathbf{C}_{1}^{\times}\right)$, and the corresponding local Heisenberg group Heis $\left(k_{\mathfrak{p}}\right)$ is the central extension of $k_{\mathfrak{p}} \oplus k_{\mathfrak{p}}^{*}$ by $\mathbf{C}_{1}^{\times}$with group law twisted by $B_{\mathfrak{p}}$.

By definition, the symplectic group (globally as well as locally) is the isotropy group for the foregoing data. It is well known (and in any case easy to prove) that in this lowestdimensional case we just get $\mathrm{SL}_{2}(k)$ and $\mathrm{SL}_{2}\left(k_{\mathfrak{p}}\right)$.

Working locally first, with Heis $\left(k_{\mathfrak{p}}\right)$ and $\mathrm{SL}_{2}\left(k_{\mathfrak{p}}\right)$, we can use the Stone-Von Neumann theorem to infer that, up to conjugation (which will presently be crucial), there exists a unique irreducible unitary representation, $\varrho$, of Heis $\left(k_{\mathfrak{p}}\right)$ in the usual associated SchwartzBruhat representation space, $S_{\mathfrak{p}}$, whose central character comes from $q_{B_{\mathfrak{p}}}$, the quadratic form defined by $B_{\mathfrak{p}}$. For $w=(z, \xi)$, where $z \in k_{\mathfrak{p}} \oplus k_{\mathfrak{p}}^{*}, \xi \in \mathbf{C}_{1}^{\times}$, and for $\sigma \in \mathrm{SL}_{2}\left(k_{\mathfrak{p}}\right)$, the natural action given by $w \mapsto w^{\sigma}:=\left(z^{\sigma}, \xi\right)$ yields an action on $\varrho$ in that we may define $\varrho^{\sigma}$ : $w \mapsto \varrho\left(w^{\sigma}\right)$. It is immediate that $\varrho^{\sigma}$ is also (with $\varrho$ ) an irreducible unitary representation of Heis $\left(k_{\mathfrak{p}}\right)$, evidently sharing $\varrho$ 's central character. So, by the essential uniqueness part of the Stone-Von Neumann theorem, $\varrho$ and $\varrho^{\sigma}$ are conjugate mappings and this provides that for every $\sigma \in \mathrm{SL}_{2}\left(k_{\mathfrak{p}}\right)$ we obtain an element $r_{\mathfrak{p}}(\sigma)$ in the automorphism group of $S_{\mathfrak{p}}$ realizing this conjugation. It follows on general algebraic grounds that, as an operator, $r_{\mathfrak{p}}$ realizes a complex projective representation, the (local) Weil representation. Projectivity 
entails that there exists a local 2-cocycle $c_{\mathfrak{p}} \in H^{2}\left(\mathrm{SL}_{2}\left(k_{\mathfrak{p}}\right), \mathbf{C}_{1}^{\times}\right)$characterized by the fact that for all $\sigma_{1}, \sigma_{2} \in \mathrm{SL}_{2}\left(k_{\mathfrak{p}}\right), r_{\mathfrak{p}}\left(\sigma_{1}\right) r_{\mathfrak{p}}\left(\sigma_{2}\right)=c_{\mathfrak{p}}\left(\sigma_{1}, \sigma_{2}\right) r_{\mathfrak{p}}\left(\sigma_{1} \sigma_{2}\right)$.

In [26], Weil shows that $c_{\mathfrak{p}}$ takes values in $\mu_{2}=\{1,-1\}$ so that $c_{\mathfrak{p}}$ actually determines a central extension, or double cover, of $\mathrm{SL}_{2}\left(k_{\mathfrak{p}}\right)$ by $\mu_{2}$ which we denote by $\mathrm{SL}_{2}\left(k_{\mathfrak{p}}\right) \times_{c_{\mathfrak{p}}} \mu_{2}$ or simply $\widetilde{S L}_{2}\left(k_{\mathfrak{p}}\right)$; in particular we have a $c_{\mathfrak{p}}$-twisted group law given by the short exact sequence (or s.e.s.)

$$
1 \longrightarrow \mu_{2} \stackrel{j}{\longrightarrow} \widetilde{S L}_{2}\left(k_{\mathfrak{p}}\right) \stackrel{p}{\longrightarrow} \mathrm{SL}_{2}\left(k_{\mathfrak{p}}\right) \longrightarrow 1,
$$

with $j$ the obvious injection and $p$ the obvious projection.

Although it was already brought out in [26] that $c_{\mathfrak{p}}$ should bear a close kinship to quadratic symbols, this was not made completely explicit until Kubota, treating the general case of $n$-fold covering of $\mathrm{SL}_{2}\left(k_{\mathfrak{p}}\right)$, presented a definition of the defining 2-cocycle in terms of the $n$-Hilbert symbol on $k_{\mathfrak{p}}^{\times} \times k_{\mathfrak{p}}^{\times}$[19]. In the present quadratic case, the local projective Weil representations $r_{\mathfrak{p}}$, with $\mathfrak{p}$ ranging over the places of $k$, admit to adelization. This is to say that there is a canonical way of defining an adelic projective representation $r_{\mathbf{A}}=\otimes_{\mathfrak{p}} r_{\mathfrak{p}}$ (in the notation of [11]; see also [17, page 52]) of the adelic group $\mathrm{SL}_{2}(k)_{\mathrm{A}}$, whose cocycle is $c_{\mathrm{A}}:=\prod_{\mathfrak{p}} c_{\mathfrak{p}}$. Accordingly we obtain the adelic covering data

$$
1 \longrightarrow \mu_{2} \stackrel{j}{\rightarrow} \widetilde{\mathrm{SL}}_{2}(k)_{\mathrm{A}}:=\mathrm{SL}_{2}(k)_{\mathbf{A}} \underset{c_{\mathrm{A}}}{\times} \mu_{2} \stackrel{p}{\longrightarrow} \mathrm{SL}_{2}(k)_{\mathrm{A}} \longrightarrow 1 .
$$

In view of Kubota's presentation of $c_{\mathfrak{p}}$ about which we say more in due course, one shows relatively easily that 2 -Hilbert reciprocity, that is,

$$
\prod_{p}\left(\frac{a, b}{\mathfrak{p}}\right)_{2}=1
$$

for all $a, b \in k^{\times}$, holds as a consequence of $\widetilde{\mathrm{SL}}_{2}(k)_{\mathrm{A}}$ (or $c_{\mathrm{A}}$ ) being split on $\mathrm{SL}_{2}(k)$ in a particularly strong sense; moreover, the converse holds, too. Of course, here $(\cdot, \cdot / \mathfrak{p})_{2}$ denotes the 2-Hilbert symbol.

In diagrammatical language, then, quadratic reciprocity comes down to

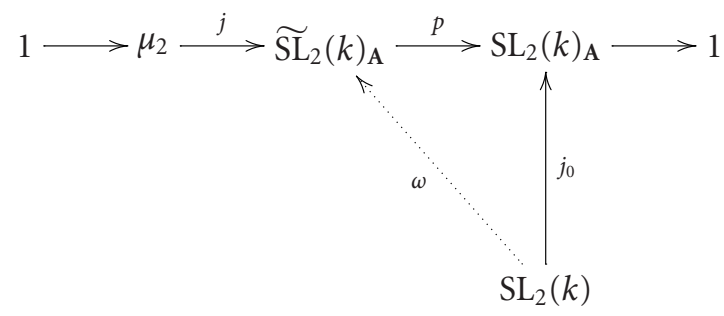

where $j_{0}$ is the diagonal imbedding, and, taking a familiar liberty with $\otimes$,

$$
\omega=\mathrm{id} \otimes s_{\mathbf{A}}: \sigma \longmapsto\left(\sigma, s_{\mathbf{A}}(\sigma)\right)
$$

with

$$
s_{\mathrm{A}}: \mathrm{SL}_{2}(k) \longrightarrow \mu_{2}
$$


a group homomorphism. That $s_{\mathrm{A}}$ should have this algebraic structure is what is meant by the aforementioned strong sense in which $\omega$ splits $c_{\mathrm{A}}$ on the rational points, $\mathrm{SL}_{2}(k)$.

Because the existence of $\omega$ can be derived from the behavior of $r_{\mathrm{A}}$ by Fourier-analytic means, Weil's observation, on [26, page 144], that this way of getting quadratic reciprocity is equivalent to Hecke's, is legitimized. Indeed, Weil defined an adelic functional, the wellknown $\Theta$-functional (capable of realizing Hecke $\vartheta$-functions under the right assignments [9, page 145]), and proved by a generalization of Poisson summation that this functional is invariant under the action of $r_{\mathrm{A}}\left(\mathrm{SL}_{2}(k)\right)$. It follows quickly that if $\sigma_{1}, \sigma_{2} \in \mathrm{SL}_{2}(k)$, then $c_{\mathbf{A}}\left(\sigma_{1}, \sigma_{2}\right)=1$, and then 2-Hilbert reciprocity (2.3) obtains by choosing $\sigma_{1}, \sigma_{2}$ adroitly.

As regards higher-degree metaplectic covers, that is, Hecke's challenge as discussed in Section 1, one can argue that it has thus far been impossible to generalize the foregoing tactics to get $n$-Hilbert reciprocity because of the current absence of higher-degree counterparts to the projective Weil representation. However, in $[19,20]$ Kubota succeeded in defining an $n$-fold cover of $\mathrm{SL}_{2}$ (and even $\mathrm{GL}_{2}$ ) by direct algebraic methods and such a cover, say, $\widetilde{S L}_{2}(k)_{\mathrm{A}}^{(n)}$, is split on $\mathrm{SL}_{2}(k)$, just as in the quadratic case; this suffices for the derivation mutatis mutandis of $n$-Hilbert reciprocity. But Kubota was forced to employ $n$ Hilbert reciprocity à priori in order to get this splitting on the rational points, so Hecke's challenge is left unmet because of circularity. As we mentioned in Section 1, it is our eventual objective to introduce a sheaf-theoretic formalism with which to address this algebraic strategy of Kubota while circumnavigating $n$-Hilbert reciprocity. The present paper, as a first step, is concerned with the topological preliminaries to this enterprise.

\section{The $n$-fold cover of $\mathrm{SL}_{2}$}

Let $n \geq 2$. In [19], to which we refer the reader for proofs and additional details, Kubota directly defined local 2-cocycles $c_{\mathfrak{p}}^{(n)} \in H^{2}\left(\mathrm{SL}_{2}\left(k_{\mathfrak{p}}\right), \mu_{n}\right)$, where $\mu_{n}$ is the group of $n$th roots of 1 (assumed from now on to live in $k$ ), as follows. Let $\sigma=\left(\begin{array}{ll}a & b \\ c & d\end{array}\right) \in \mathrm{SL}_{2}\left(k_{\mathfrak{p}}\right)$, set

$$
x(\sigma)= \begin{cases}c & \text { if } c \neq 0 \\ d & \text { if } c=0\end{cases}
$$

and then define, for all $\sigma_{1}, \sigma_{2} \in \mathrm{SL}_{2}\left(k_{\mathfrak{p}}\right)$,

$$
c_{\mathfrak{p}}^{(n)}\left(\sigma_{1}, \sigma_{2}\right)=\left(\frac{x\left(\sigma_{1}\right), x\left(\sigma_{2}\right)}{\mathfrak{p}}\right)_{n}\left(\frac{-x\left(\sigma_{1}\right)^{-1} x\left(\sigma_{2}\right), x\left(\sigma_{1} \sigma_{2}\right)}{\mathfrak{p}}\right)_{n},
$$

where $(\cdot, \cdot / \mathfrak{p})_{n}$ is the $n$-Hilbert symbol on $k_{\mathfrak{p}}^{\times} \times k_{\mathfrak{p}}^{\times}$. The principal result in [19] that $c_{\mathfrak{p}}^{(n)}$ is a factor set as indicated follows entirely from local properties of the $n$-Hilbert symbol. We obtain the s.e.s.

$$
1 \longrightarrow \mu_{n} \stackrel{j}{\longrightarrow} \widetilde{\mathrm{SL}}_{2}\left(k_{\mathfrak{p}}\right)^{(n)}:=\mathrm{SL}_{2}\left(k_{\mathfrak{p}}\right) \underset{c_{\mathfrak{p}}^{(n)}}{\times} c_{\mathfrak{p}}^{(n)} \mu_{n} \stackrel{p}{\longrightarrow} \mathrm{SL}_{2}\left(k_{\mathfrak{p}}\right) \longrightarrow 1
$$

generalizing (2.1). 
It is pointed out on [20, page 22] that $n$-Hilbert reciprocity is not needed to get the adelization of (3.3) which proceeds thus: let $\sigma_{1}, \sigma_{2}$ be two adeles in $\mathrm{SL}_{2}(k)_{\mathrm{A}}$, that is, $\sigma_{1}=$ $\left(\sigma_{1, \mathfrak{p}}\right)_{\mathfrak{p}}, \sigma_{2}=\left(\sigma_{2, \mathfrak{p}}\right)_{\mathfrak{p}}$ (with each "valuation vector" having almost all coordinates in the corresponding $O_{\mathfrak{p}}$ 's), so that $c_{\mathfrak{p}}^{(n)}\left(\sigma_{1, \mathfrak{p}}, \sigma_{2, \mathfrak{p}}\right)$ is well defined for every $\mathfrak{p}$. Writing $c_{\mathfrak{p}}^{(n)}\left(\sigma_{1}, \sigma_{2}\right)$ for $c_{\mathfrak{p}}^{(n)}\left(\sigma_{1, \mathfrak{p}}, \sigma_{2, \mathfrak{p}}\right)$, we get (easily) that $c_{\mathfrak{p}}^{(n)}\left(\sigma_{1}, \sigma_{2}\right)=1$ a.e. $\mathfrak{p}$, whence we can define

$$
c_{\mathbf{A}}^{(n)}\left(\sigma_{1}, \sigma_{2}\right)=\prod_{\mathfrak{p}} c_{\mathfrak{p}}^{(n)}\left(\sigma_{1}, \sigma_{2}\right)
$$

for any $\sigma_{1}, \sigma_{2} \in \mathrm{SL}_{2}(k)_{\mathrm{A}}$. With $c_{\mathrm{A}}^{(n)} \in H^{2}\left(\mathrm{SL}_{2}(k)_{\mathrm{A}}, \mu_{n}\right)$ in this way we immediately obtain the adelic cover

$$
1 \longrightarrow \mu_{n} \stackrel{j}{\longrightarrow} \widetilde{\mathrm{SL}}_{2}(k)_{\mathrm{A}}^{(n)}:=\mathrm{SL}_{2}(k)_{\mathbf{A}} \underset{c_{\mathrm{A}}^{(n)}}{\times} \mu_{n} \stackrel{k}{\longrightarrow} \mathrm{SL}_{2}(k)_{\mathbf{A}} \longrightarrow 1
$$

By the way, as already alluded to at the end of Section 1, another characterization of $\widetilde{\mathrm{SL}}(k)_{\mathrm{A}}^{(n)}$ can be gleaned from Matsumoto's construction [17, 21]. Moreover, Kazhdan and Patterson take special care to mention on [17, page 51] that $n$-Hilbert reciprocity is equivalent to the fact that $c_{\mathrm{A}}^{(n)}$ should be split on $\mathrm{SL}_{2}(k)$, evidently along the same lines as (2.4) (although they phrase things in terms of $\mathrm{GL}_{2}$ ). For our aims, the point is that $n$-Hilbert reciprocity is not necessary for the construction of (3.4) and (3.5), even though it is in fact sufficient (as Kubota suggests on [19, page 115]).

The upshot is that, concerning Hecke's challenge, it will be enough to devise a splitting homomorphism $\omega=\mathrm{id} \otimes s_{\mathrm{A}}$, with $s_{\mathrm{A}}: \mathrm{SL}_{2}(k) \rightarrow \mu_{n}$ (just as in (2.5) and (2.6)) situated as follows:

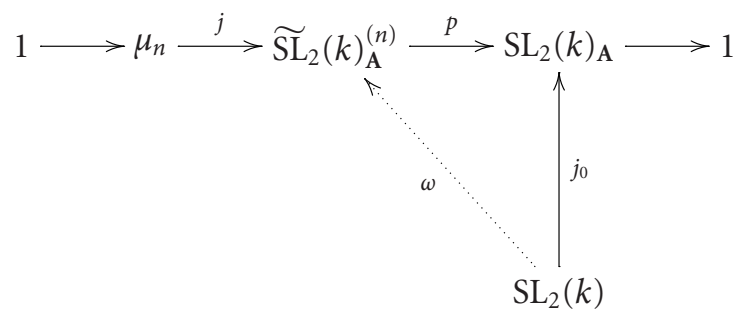

just as in (2.4). In $[19,20]$ Kubota, presupposing $n$-Hilbert reciprocity, gives an explicit formula for $s_{\mathrm{A}}$ which, in fact, provides that it is a homomorphism on $\mathrm{SL}_{2}(k)$ (see also [10, page 27]). This is the strong sense in which $\omega$ splits $c_{\mathrm{A}}^{(n)}$ on $\mathrm{SL}_{2}(k)$ as discussed above, seeing that simply because, by virtue of splitting, $s_{\mathbf{A}}\left(\sigma, \sigma_{2}\right)=c_{\mathbf{A}}^{(n)}\left(\sigma_{1}, \sigma_{2}\right)$ $s_{\mathbf{A}}\left(\sigma_{1}\right) s_{\mathbf{A}}\left(\sigma_{2}\right)$ for all $\sigma_{1}, \sigma_{2} \in \mathrm{SL}_{2}(k)$; it follows from $s_{\mathbf{A}}$ being a homomorphism that $c_{\mathbf{A}}^{(n)} \equiv 1$ on $\mathrm{SL}_{2}(k)^{2}=\mathrm{SL}_{2}(k) \times \mathrm{SL}_{2}(k)$. 
So, by way of a summary, the $n$-fold cover, $\widetilde{S L}_{2}(k)_{\mathrm{A}}^{(n)}$, defined by $c_{\mathrm{A}}^{(n)}$ in (3.4), does not require $n$-Hilbert reciprocity, that is, for all $a, b \in k^{\times}$

$$
\prod_{\mathfrak{p}}\left(\frac{a, b}{\mathfrak{p}}\right)_{n}=1,
$$

for its existence; but $n$-Hilbert reciprocity is equivalent to $c_{\mathrm{A}}^{(n)}$ being split on $\mathrm{SL}_{2}(k)$, the set of rational points of $\mathrm{SL}_{2}(k)_{\mathrm{A}}$, by $\omega$, as per (3.6). This means that

$$
p \circ \omega=j_{0},
$$

in the indicated strong sense that

$$
\omega=\mathrm{id} \otimes s_{\mathrm{A}}
$$

where

$$
s_{\mathrm{A}}: \mathrm{SL}_{2}(k) \longrightarrow \mu_{n}
$$

is a homomorphism (i.e., $c_{\mathrm{A}}^{(n)} \equiv 1$ on $\left.\mathrm{SL}_{2}(k)^{2}\right)$. Accordingly we now focus our attention on demonstrating the existence of $\omega$ and $s_{\mathrm{A}}$ by means of derived and triangulated categories and perverse sheaves.

\section{Topological groups and stratifications}

The respective topological groups underlying $\widetilde{\mathrm{SL}}_{2}\left(k_{\mathfrak{p}}\right)^{(n)}$ and $\widetilde{\mathrm{SL}}_{2}(k)_{\mathrm{A}}^{(n)}$, as per $(3.3)$ and (3.5), are just $\mathrm{SL}_{2}\left(k_{\mathfrak{p}}\right) \times \mu_{n}$ and $\mathrm{SL}_{2}(k)_{\mathrm{A}} \times \mu_{n}$ with the obvious product topologies. We will provide an example immediately to show that in general $c_{\mathfrak{p}}^{(n)}$, as given by (3.1) and (3.2), fails to be continuous, whence the $c_{\mathfrak{p}}^{(n)}$-twisted group structure on $\widetilde{S L}_{2}\left(k_{\mathfrak{p}}\right)^{(n)}$ fails to realize this group as an autonomous topological group. This forces us to approach the matter of topologically encoding this twisting in a new way, as we will see presently. But let us first take a look at our example.

Example 4.1. Let $\mathfrak{p}=\infty, \sigma_{m}=\left(\begin{array}{cc}1+1 / m & 1 \\ 1 / m & 1\end{array}\right)$. Then, as $m \rightarrow \infty, \sigma_{m} \rightarrow\left(\begin{array}{ll}1 & 1 \\ 0 & 1\end{array}\right)$ so that $($ by $(3.1))$ we get $x\left(\lim _{m \rightarrow \infty} \sigma_{m}\right)=x\left(\left(\begin{array}{ll}1 & 1 \\ 0 & 1\end{array}\right)\right)=1$ while, from $x\left(\sigma_{m}\right)=1 / m$, it follows that $\lim _{m \rightarrow \infty} x\left(\sigma_{m}\right)=$ 0 . So, $x$ is discontinuous. Coupled with the continuity of the $n$-Hilbert symbol (at all places; see [18, page 101]) this compromises the continuity of $c_{\infty}^{(n)}$.

On to the topological maneuvers. We work in the adelic topology, noting that, except for the splitting in (3.6), everything goes through in exactly the same way in the $\mathfrak{p}$-adic case. The following sequence of topological groups is obviously split exact:

$$
1 \longrightarrow \mu_{n} \stackrel{j}{\longrightarrow} \mathrm{SL}_{2}(k)_{\mathbf{A}} \times \mu_{n} \stackrel{p}{\longrightarrow} \mathrm{SL}_{2}(k)_{\mathbf{A}} \longrightarrow 1
$$

Double it in the following sense (generally writing $X^{2}$ for $X \times X$, as done above already for $\left.\mathrm{SL}_{2}(k)\right)$ :

$$
\mu_{n}^{2} \stackrel{j \otimes j}{\longrightarrow}\left(\mathrm{SL}_{2}(k)_{\mathrm{A}} \times \mu_{n}\right)^{2} \stackrel{p \otimes p}{\longrightarrow} \mathrm{SL}_{2}(k)_{\mathrm{A}}^{2}
$$


where we use the alternative notation, $\longrightarrow, \rightarrow$, for injections and surjections to distinguish that we are working in the category of topological spaces as opposed to the category of groups: (4.1) and (3.5) live in the category of (topological) groups, but (4.2) lives in the category of topological spaces. We also take the notational liberty of writing, generally, $f \otimes g:(x, y) \mapsto(f(x), f(y))$. Next, write $m_{\ell}$ (resp., $m_{r}$ ) for multiplication in $\mu_{n}$ (resp., $\left.\mathrm{SL}_{2}(k)_{\mathrm{A}}\right)$ and write $m_{c_{\mathrm{A}}^{(n)}}$ for the $c_{\mathrm{A}}^{(n)}$-twisted multiplication in $\widetilde{\mathrm{SL}}(k)_{\mathrm{A}}^{(n)}$; obtain the hybridized diagram

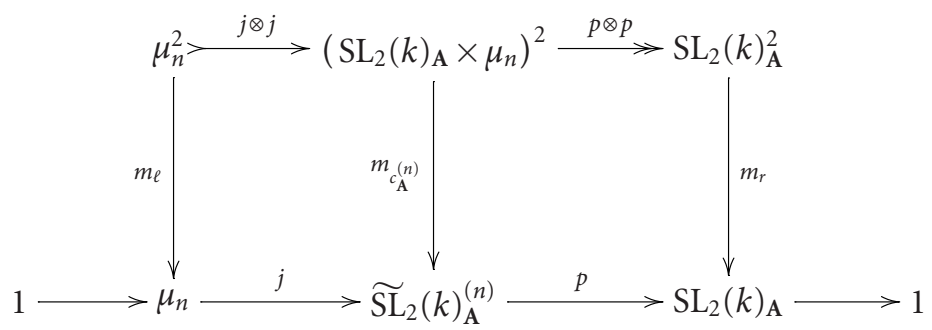

Now, in $\mathrm{SL}_{2}(k)_{\mathrm{A}}^{2}$, say $\left(\sigma_{1}, \sigma_{2}\right) \sim\left(\sigma_{1}^{\prime}, \sigma_{2}^{\prime}\right)$ if and only if $c_{\mathrm{A}}^{(n)}\left(\sigma_{1}, \sigma_{2}\right)=c_{\mathrm{A}}^{(n)}\left(\sigma_{1}^{\prime}, \sigma_{2}^{\prime}\right)$, an obvious equivalence relation whose equivalence classes are precisely the sets $\left(c_{\mathrm{A}}^{(n)}\right)^{-1}(\xi)$, with $\xi \in \mu_{n}$. This equivalence relation lifts immediately to $\left(\mathrm{SL}_{2}(k)_{\mathrm{A}} \times \mu_{n}\right)^{2}$ by setting $\left(\left(\sigma_{1}, \xi_{1}\right),\left(\sigma_{2}, \xi_{2}\right)\right) \sim\left(\left(\sigma_{1}^{\prime}, \xi_{1}^{\prime}\right),\left(\sigma_{2}^{\prime}, \xi_{2}^{\prime}\right)\right)$ if and only if, again, $c_{\mathbf{A}}^{(n)}\left(\sigma_{1}, \sigma_{2}\right)=c_{\mathbf{A}}^{(n)}\left(\sigma_{1}^{\prime}, \sigma_{2}^{\prime}\right)$; get equivalence classes of the form $\left(c_{\mathrm{A}}^{(n)}\right)^{-1}(\xi) \times \mu_{n}^{2}$, with a harmless abuse of notation (identifying $\left(\left(\sigma_{1}, \xi_{1}\right),\left(\sigma_{2}, \xi_{2}\right)\right)$ with $\left.\left(\sigma_{1}, \sigma_{2}, \xi_{1}, \xi_{2}\right)\right)$. Observe that if $c_{\mathbf{A}}^{(n)}\left(\sigma_{1}, \sigma_{2}\right)=c_{\mathbf{A}}^{(n)}\left(\sigma_{1}^{\prime}, \sigma_{2}^{\prime}\right)=\xi_{0}$, then, in $\widetilde{\mathrm{SL}}_{2}(k)_{\mathrm{A}}^{(n)}$, the products $\left(\sigma_{1}, \xi_{1}\right)\left(\sigma_{2}, \xi_{2}\right)$ and $\left(\sigma_{1}^{\prime}, \xi_{1}^{\prime}\right)\left(\sigma_{2}^{\prime}, \xi_{2}^{\prime}\right)$, with $\xi_{1}, \xi_{2}, \xi_{1}^{\prime}, \xi_{2}^{\prime}$ unrestricted, each obtain as multiplicative translations by $\xi_{0}$ in the second coordinate of, respectively, $\left(\sigma_{1} \sigma_{2}, \xi_{1} \xi_{2}\right)$ and $\left(\sigma_{1}^{\prime} \sigma_{2}^{\prime}, \xi_{1}^{\prime} \xi_{2}^{\prime}\right)$, both in $\mathrm{SL}_{2}(k)_{\mathbf{A}} \times \mu_{n}$. Therefore, the given equivalence relation provides a way of encoding the action of twisting by $c_{\mathrm{A}}^{(n)}$ in the setting of $\left(\mathrm{SL}_{2}(k)_{\mathrm{A}} \times \mu_{n}\right)^{2}$, partitioned into the equivalence classes $\left(c_{\mathrm{A}}^{(n)}\right)^{-1}(\xi) \times \mu_{n}^{2}$, with $\xi$ ranging over $\mu_{n}$ : for a given choice, $\xi=\xi_{0}$, the effect of $m_{c_{\mathrm{A}}^{(n)}}$ on the class of $\xi_{0}$ is to append the aforementioned translation by $\xi_{0}$ to the untwisted result of multiplying in $\mathrm{SL}_{2}(k)_{\mathbf{A}} \times \mu_{n}$. In order to bring this out diagrammatically we recast (4.3) as follows, bearing in mind that the earlier harmless abuse of notation is still in effect (and will be from now on):

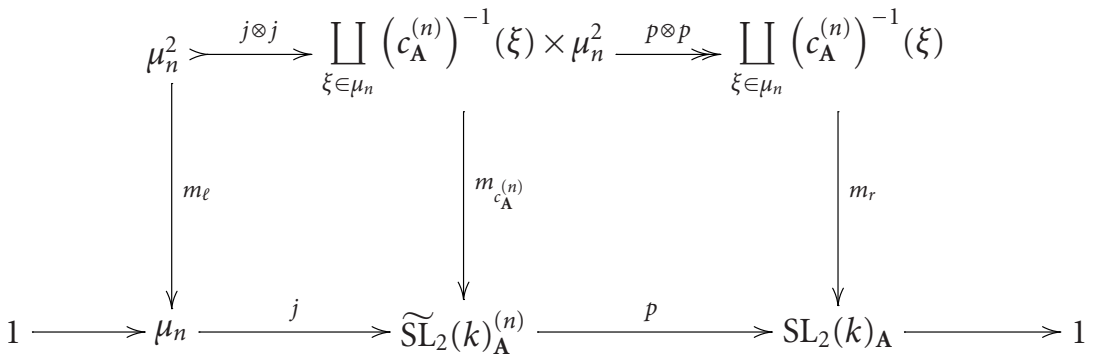

We reiterate that $\left(\sigma_{1}, \sigma_{2}, \xi_{1}, \xi_{2}\right) \in\left(c_{\mathbf{A}}^{(n)}\right)^{-1}\left(\xi_{0}\right) \times \mu_{n}^{2}$ is tantamount to having $c_{\mathbf{A}}^{(n)}\left(\sigma_{1}, \sigma_{2}\right)=\xi_{0}$ and the effect of $m_{c_{A}^{(n)}}$ on the element is to map it to $\left(\sigma_{1}, \sigma_{2}, \xi_{1} \xi_{2} \xi_{0}\right)$. 
In the setting of (4.4) the splitting of $c_{\mathrm{A}}^{(n)}$ by $\omega$ as discussed at the end of Section 3 takes the following shape. We have seen that, in order to get at Hecke's challenge in accord with [19], it is necessary and sufficient that $c_{\mathbf{A}}^{(n)}\left(\sigma_{1}, \sigma_{2}\right)=1$ for all $\sigma_{1}, \sigma_{2} \in \mathrm{SL}_{2}(k)$, that is, $\mathrm{SL}_{2}(k)^{2} \subset\left(c_{\mathbf{A}}^{(n)}\right)^{-1}(1)$. Said differently, we require a mapping

$$
\Omega: \mathrm{SL}_{2}(k)^{2} \longrightarrow\left(\mathrm{SL}_{2}(k)_{\mathbf{A}} \times \mu_{n}\right)^{2}=\coprod_{\xi \in \mu_{n}}\left(c_{\mathbf{A}}^{(n)}\right)^{-1}(\xi) \times \mu_{n}^{2}
$$

such that

$$
\operatorname{im}(\Omega) \subset\left(c_{\mathrm{A}}^{(n)}\right)^{-1}(1) \times \mu_{n}^{2}
$$

and, with $m_{0}: \mathrm{SL}_{2}(k)^{2} \rightarrow \mathrm{SL}_{2}(k)$ the usual group law on $\mathrm{SL}_{2}(k)$, we want that

$$
\omega \circ m_{0}=m_{c_{\mathrm{A}}^{(n)}} \circ \Omega
$$

It is enough to prove that (4.6) holds; (4.7) is immediate (see (4.8) below). The hard part is getting $\Omega$ and $\omega$ so that the latter splits $c_{\mathrm{A}}^{(n)}$ on $\mathrm{SL}_{2}(k)$, which is a somewhat different matter, as we will see momentarily.

As we have already indicated a few times, the strategy we seek to employ in attacking (4.6) is, in broad terms, concerned with employing the machinery of derived and triangulated categories and perverse sheaves. Soon we turn to the topological prerequisites for this approach; the algebraic situation is summarized in the following diagram extending (4.4):

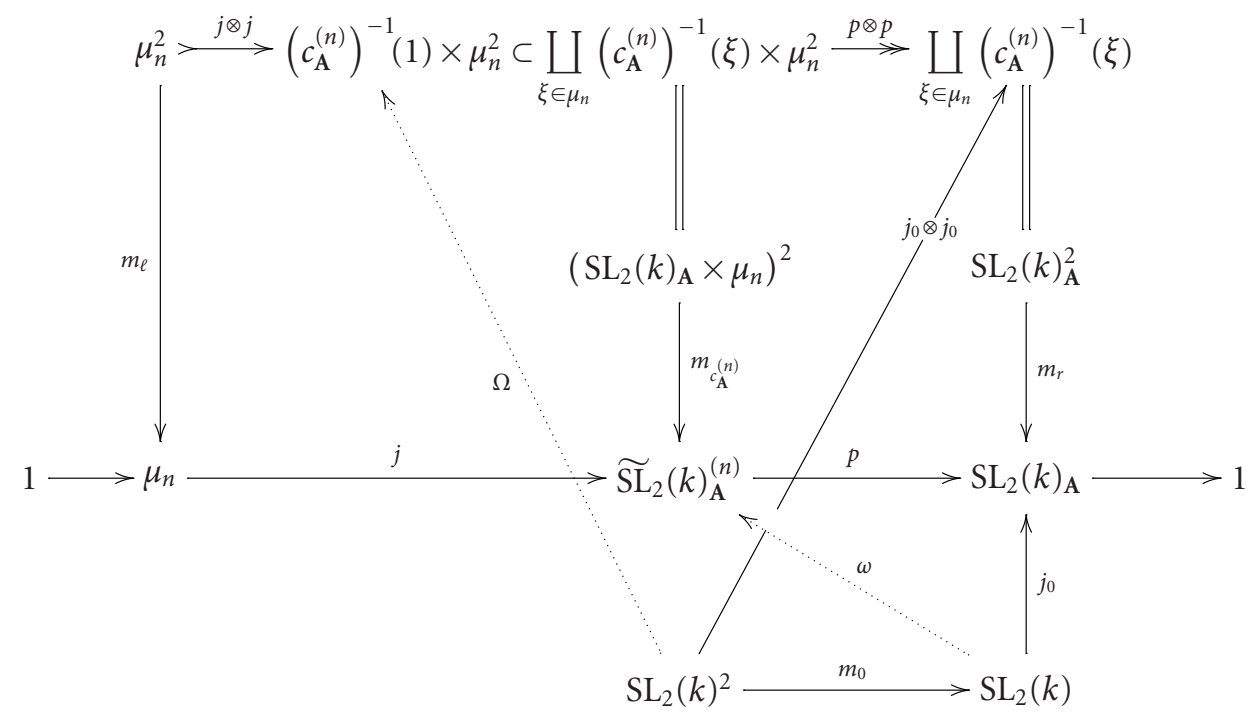


Here the critical subdiagram is, obviously,

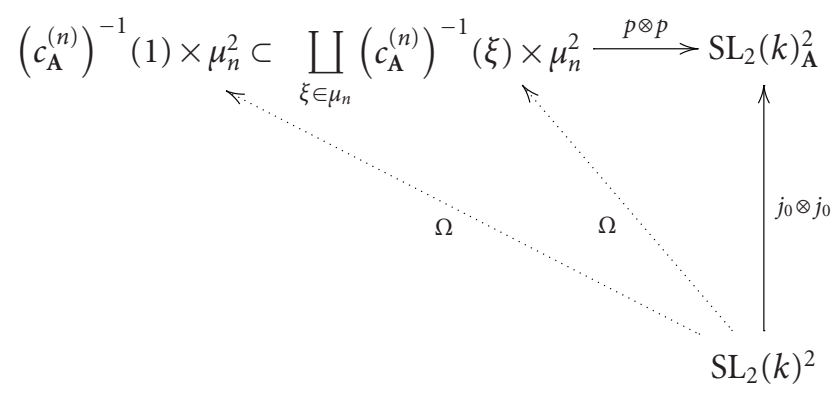

where the notational abuse of duplicating $\Omega$ is justified à forteriori once (4.6) is taken care of. Note, too, that the requirement $p \circ \omega=j_{0}$ (see (3.8)) is equivalent to having $(p \otimes p) \circ \Omega=j_{0} \otimes j_{0}$.

Now, as regards the topological prerequisites just alluded to, we need first of all to address the question of stratification (in the sense of [1] of course). Starting with the local case we have the following proposition.

Proposition 4.2. Each set $\left(c_{\mathfrak{p}}^{(n)}\right)^{-1}(\xi)$ is locally closed in $\mathrm{SL}_{2}\left(k_{\mathfrak{p}}\right)^{2}$.

Proof. With $c_{\mathfrak{p}}^{(n)}$ given by (3.2) we get that, with $\sigma_{1}, \sigma_{2} \in \mathrm{SL}_{2}\left(k_{\mathfrak{p}}\right)$,

$$
\begin{aligned}
& \left(c_{\mathfrak{p}}^{(n)}\right)^{-1}(\xi) \\
& \quad=\coprod_{\nu \in \mu_{n}}\left\{\left(\sigma_{1}, \sigma_{2}\right) \mid\left(\frac{x\left(\sigma_{1}\right), x\left(\sigma_{2}\right)}{\mathfrak{p}}\right)_{n}=v,\left(\frac{-x\left(\sigma_{1}\right)^{-1} x\left(\sigma_{2}\right), x\left(\sigma_{1} \sigma_{2}\right)}{\mathfrak{p}}\right)_{n}=\xi \nu^{-1}\right\} .
\end{aligned}
$$

Write $\sigma_{1}=\left(\begin{array}{ll}a & b \\ c & d\end{array}\right), \sigma_{2}=\left(\begin{array}{ll}e & f \\ g & h\end{array}\right)$, so that $\sigma_{1} \sigma_{2}=\left(\begin{array}{ll}a e+b g & a f+b h \\ c e+d g & c f+d h\end{array}\right)$, and distinguish the following four sets partitioning $\mathrm{SL}_{2}\left(k_{\mathfrak{p}}\right)^{2}$ :

(i) $A=\left\{\left(\sigma_{1}, \sigma_{2}\right) \mid c, g \neq 0\right\}$,

(ii) $B=\left\{\left(\sigma_{1}, \sigma_{2}\right) \mid c=0, g \neq 0\right\}$,

(iii) $C=\left\{\left(\sigma_{1}, \sigma_{2}\right) \mid c \neq 0, g=0\right\}$,

(iv) $D=\left\{\left(\sigma_{1}, \sigma_{2}\right) \mid c=0, d=0\right\}$.

Infer that if $\left(\sigma_{1}, \sigma_{2}\right) \in A$, then $x\left(\sigma_{1}\right)=c$, and $x\left(\sigma_{2}\right)=g$; if $\left(\sigma_{1}, \sigma_{2}\right) \in B$, then $x\left(\sigma_{1}\right)=d$ and $x\left(\sigma_{2}\right)=g$; if $\left(\sigma_{1}, \sigma_{2}\right) \in C$, then $x\left(\sigma_{1}\right)=c, x\left(\sigma_{2}\right)=h$; and if $\left(\sigma_{1}, \sigma_{2}\right) \in D$, then $x\left(\sigma_{1}\right)=d$, $x\left(\sigma_{2}\right)=h$. Accordingly each consituent set in (4.10) (for every $v \in \mu_{n}$ ) is partitioned in turn into the disjoint union of four subsets of $A, B, C, D$, say, $A_{\nu}, B_{v}, C_{\gamma}, D_{v}$, defined by the preceding conditions. Given that we now have $4 n$ pairwise disjoint sets, that is, finitely many, it is enough to check that each of these is locally closed. Consider, for a fixed $\nu$, the (typical) set $A_{v}$

$$
A_{\nu}=\left\{\left(\sigma_{1}, \sigma_{2}\right) \in A \mid\left(\frac{c, g}{\mathfrak{p}}\right)_{n}=\nu,\left(\frac{-g / c, x\left(\sigma_{1}, \sigma_{2}\right)}{\mathfrak{p}}\right)=\xi \nu^{-1}\right\}
$$


Regarding $x\left(\sigma_{1} \sigma_{2}\right)$ we get that $c e+d g=0$ yields either that $d, e \neq 0$ or $d=e=0$; whence, as $A$ (and so $A_{\nu}$ ) is characterized by $c, g \neq 0$, the latter condition, $d=e=0$, forces $b=$ $-1 / c, g=-1 / f$. However, this is not really of concern to us: what does matter is that $A_{\gamma}$, in turn, is partitioned into the disjoint union of three sets as follows:

$$
\begin{aligned}
A_{v}= & \left\{\left(\sigma_{1}, \sigma_{2}\right) \mid c e+d g \neq 0,\left(\frac{c, g}{\mathfrak{p}}\right)_{n}=v,\left(\frac{-g / c, c e+d g}{\mathfrak{p}}\right)_{n}=\xi \nu^{-1}\right\} \\
& \amalg\left\{\left(\sigma_{1}, \sigma_{2}\right) \mid c e+d g=0, \text { with } d=e=0,\left(\frac{c, g}{\mathfrak{p}}\right)_{n}=v,\left(\frac{-g / c, c f+d h}{\mathfrak{p}}\right)_{n}=\xi v^{-1}\right\} \\
& \amalg\left\{\left(\sigma_{1}, \sigma_{2}\right) \mid c e+d g=0, \text { with } d=e=0,\left(\frac{c, g}{\mathfrak{p}}\right)_{n}=v,\left(\frac{-g / c, c f+d h}{\mathfrak{p}}\right)_{n}=\xi v^{-1}\right\} .
\end{aligned}
$$

Consider, for example, the second set in (4.12), cut out by the conditions $c, d, e, g \neq 0$, $c e+d g=0,((c, g) / \mathfrak{p})_{n}=v,((-g / c, c f+d h) / \mathfrak{p})_{n}=\xi \nu^{-1}$, making for the intersection of seven (obvious) sets. By the continuity of the ordinary arithmetical operations, the equation $c e+d g=0$ cuts out a closed set; the conditions $c, d, e, g \neq 0$ cut out four open sets. Beyond this we have that it follows from local class field theory that the $n$-Hilbert symbol is continuous (as we had occasion to note above, in our example concerning the discontinuity of $\left.c_{\infty}^{(n)}\right)$; whence, with $-g / c, c f+d h$ continuous, too, the remaining conditions, $((c, g) / \mathfrak{p})_{n}=\nu$ and $(-(g / c, c f+d h) / \mathfrak{p})_{n}=\xi \nu^{-1}$ cut out closed sets. It follows immediately that the second set in (4.12) is locally closed. The same kinds of arguments conspire to prove the other two sets in (4.12) to be locally closed. So $A_{v}$ is locally closed. Since the decomposition (4.12) of $A_{\nu}$ is altogether typical vis à vis its counterparts $B_{v}, C_{v}$, and $D_{v}$, we may conclude that, similarly, each of these is locally closed. Since $\nu \in \mu_{n}$, a finite set, get that $A, B, C, D$, and, therefore, $\left(c_{\mathfrak{p}}^{(n)}\right)^{-1}(\xi)$, as per (4.10), are locally closed.

Corollary 4.3. Each set $\left(c_{\mathfrak{p}}^{(n)}\right)^{-1}(\xi) \times \mu_{n}^{2}$ is locally closed in $\left(\mathrm{SL}_{2}\left(k_{\mathfrak{p}}\right) \times \mu_{n}\right)^{2}$.

Proof. Being open as well as being closed commute with passing to a product topology relative to a discrete space.

Turning now to the adelic case, consider the equation

$$
c_{\mathrm{A}}^{(n)}\left(\sigma_{1}, \sigma_{2}\right)=\prod_{\mathfrak{p}} c_{\mathfrak{p}}^{(n)}\left(\sigma_{1, \mathfrak{p}}, \sigma_{2, \mathfrak{p}}\right)=\xi
$$

where $\sigma_{1}=\left(\sigma_{1, \mathfrak{p}}\right)_{\mathfrak{p}}, \sigma_{2}=\left(\sigma_{2, \mathfrak{p}}\right)_{\mathfrak{p}} \in \mathrm{SL}_{2}\left(k_{\mathfrak{p}}\right)$, as before, and $\xi \in \mu_{n}$. Fixing an ordering of the places $\mathfrak{p}$ of $k$ we define a multiplicative partition, $\pi_{\ell}^{\times}(\xi)$, of length $\ell$ (which we may suppress, writing $\pi^{\times}(\xi)$ ), to be any adele $\left(\alpha_{\mathfrak{p}}\right)_{\mathfrak{p}}$ such that in the $\ell$ th position $\alpha_{\mathfrak{p}} \neq 1$ but in all subsequent places $\alpha_{\mathfrak{p}}=1$ (for $\pi^{\times}(\xi)$ such an $\ell$ should exist), and, additionally,

$$
\prod_{\mathfrak{p}} \alpha_{\mathfrak{p}}=\xi
$$


It follows immediately that (4.13) holds if and only if $c_{\mathfrak{p}}^{(n)}\left(\sigma_{1, \mathfrak{p}} \sigma_{2, \mathfrak{p}}\right)=\alpha_{\mathfrak{p}}$ for all $\mathfrak{p}$, for some $\pi^{\times}(\xi)$; in other words, we have that $\left(\sigma_{1}, \sigma_{2}\right)=\left(\left(\sigma_{1, \mathfrak{p}}\right)_{\mathfrak{p}},\left(\sigma_{2, \mathfrak{p}}\right)_{\mathfrak{p}}\right) \in\left(c_{\mathbf{A}}^{(n)}\right)^{-1}(\xi)$ if and only if there exists a multiplicative partition $\pi^{\times}(\xi)=\left(\alpha_{\mathfrak{p}}\right)_{\mathfrak{p}}$, of $\xi$, such that, for any $\mathfrak{p},\left(\sigma_{1, \mathfrak{p}}\right.$, $\left.\sigma_{2, \mathfrak{p}}\right) \in\left(c_{\mathfrak{p}}^{(n)}\right)^{-1}\left(\alpha_{\mathfrak{p}}\right)$.

And now we get the following proposition.

Proposition 4.4. If $\left(c_{\mathbf{A}}^{(n)}\right)^{-1}\left(\xi ; \pi^{\times}(\xi)\right)$ is the set of all pairs of adeles $\left(\sigma_{1}, \sigma_{2}\right)=\left(\left(\sigma_{1, \mathfrak{p}}\right)_{\mathfrak{p}}\right.$, $\left.\left(\sigma_{2, \mathfrak{p}}\right)_{\mathfrak{p}}\right) \in \mathrm{SL}_{2}(k)_{\mathbf{A}}^{2}$ such that $c_{\mathbf{A}}^{(n)}\left(\sigma_{1}, \sigma_{2}\right)=\xi$ in that $c_{\mathfrak{p}}^{(n)}\left(\sigma_{1, \mathfrak{p}}, \sigma_{2, \mathfrak{p}}\right)=\alpha_{\mathfrak{p}}$, where $\pi^{\times}(\xi)=$ $\left(\alpha_{\mathfrak{p}}\right)_{\mathfrak{p}}$, then $\left(c_{\mathbf{A}}^{(n)}\right)^{-1}\left(\xi ; \pi^{\times}(\xi)\right)$ is locally closed.

Proof. Write $S_{\pi^{\times}(\xi)}$ for the set of $\ell$ places (with $\pi^{\times}(\xi)$ of length $\ell$ ) $\mathfrak{p}_{1}, \mathfrak{p}_{2}, \ldots, \mathfrak{p}_{\ell}$ for which, possibly, $\alpha_{\mathfrak{p}_{i}} \neq 1$, that is, the initial segment of the ordered set of places of $k$, outside of which the coordinates $\alpha_{\mathfrak{p}}$ occupy $O_{\mathfrak{p}}$. We can form the restricted product

$$
\prod_{\mathfrak{p} \in S_{\pi^{\times}(\xi)}}\left(c_{\mathfrak{p}}^{(n)}\right)^{-1}\left(\alpha_{\mathfrak{p}}\right) \times \prod_{\mathfrak{p} \notin S_{\pi^{\times}(\xi)}}\left(c_{\mathfrak{p}}^{(n)}\right)^{-1}(1) \cap \mathrm{SL}_{2}(k)_{\mathbf{A}}^{2}
$$

and obtain herein the collection of all adele pairs $\left(\sigma_{1}, \sigma_{2}\right)$ which are mapped to $\xi$ by $c_{\mathrm{A}}^{(n)}=\prod_{\mathfrak{p}} c_{\mathfrak{p}}^{(n)}$ by means of $\pi^{\times}(\xi)$. In other words, (4.15) defines nothing else than $\left(c_{\mathrm{A}}^{(n)}\right)^{-1}\left(\xi ; \pi^{\times}(\xi)\right)$. Now, by means of Proposition 4.2 , every factor in (4.15) is $\mathfrak{p}$-adically locally closed so that there must exist open sets $U_{1}^{(\mathfrak{p})}, U_{\alpha_{\mathfrak{p}}}^{(\mathfrak{p})}$ and closed sets $F_{1}^{(\mathfrak{p})}, F_{\alpha_{\mathfrak{p}}}^{(\mathfrak{p})}$ for which $\left(c_{\mathfrak{p}}^{(n)}\right)^{-1}\left(\alpha_{\mathfrak{p}}\right)=U_{\alpha_{\mathfrak{p}}}^{(\mathfrak{p})} \cap F_{\alpha_{\mathfrak{p}}}^{(\mathfrak{p})},\left(c_{\mathfrak{p}}^{(n)}\right)^{-1}(1)=U_{1}^{(\mathfrak{p})} \cap F_{1}^{(\mathfrak{p})}$. Consequently,

$$
\begin{aligned}
\left(c_{\mathbf{A}}^{(n)}\right)^{-1}\left(\xi ; \pi^{\times}(\xi)\right)= & {\left[\prod_{\mathfrak{p} \in S_{\pi^{\times}(\xi)}} U_{\alpha_{\mathfrak{p}}}^{(\mathfrak{p})} \times \prod_{\mathfrak{p} \notin S_{\pi^{\times}(\xi)}} U_{1}^{(\mathfrak{p})}\right] } \\
& \cap\left[\prod_{\mathfrak{p} \in S_{\pi^{\times}(\xi)}} F_{\alpha_{\mathfrak{p}}}^{(\mathfrak{p})} \times \prod_{\mathfrak{p} \notin S_{\pi^{\times}(\xi)}} F_{1}^{(\mathfrak{p})}\right] \cap \mathrm{SL}_{2}(k)_{\mathbf{A}}^{2}
\end{aligned}
$$

upon rearranging the Cartesian product (generalizing the set-theoretic relation that $\left.\left(U_{1} \cap F_{1}\right) \times\left(U_{2} \times F_{2}\right)=\left(U_{1} \times U_{2}\right) \cap\left(F_{1} \times F_{2}\right)\right)$. Infer from $(4.16)$ that $\left(c_{\mathrm{A}}^{(n)}\right)^{-1}\left(\xi ; \pi^{\times}(\xi)\right)$ is adelically locally closed.

COROLlary 4.5. For every $\xi \in \mu_{n}$ and every multiplicative partition $\pi^{\times}(\xi)$, of $\xi$, the set $\left(c_{\mathrm{A}}^{(n)}\right)^{-1}\left(\xi ; \pi^{\times}(\xi)\right) \times \mu_{n}^{2} \subset\left(\mathrm{SL}_{2}(k)_{\mathbf{A}} \times \mu_{n}\right)^{2}$ is locally closed.

Proof. This is similar to the proof of Corollary 4.3 .

COROLlary 4.6. For every $\xi \in \mu_{n}$, we have that $\left(c_{\mathrm{A}}^{(n)}\right)^{-1}(\xi) \times \mu_{n}^{2}=\coprod_{\text {all } \pi^{\times}(\xi)}\left(c_{\mathrm{A}}^{(n)}\right)^{-1}(\xi$; $\left.\pi^{\times}(\xi)\right) \times \mu_{n}^{2}$. Furthermore, if we write $\ell\left(\pi^{\times}(\xi)\right.$ ) for the length of $\pi^{\times}(\xi)$ (so that $\ell\left(\pi_{\ell}^{\times}(\xi)\right.$ ) $=\ell$ ), we obtain that

$$
\left(c_{\mathrm{A}}^{(n)}\right)^{-1}(\xi) \times \mu_{n}^{2}=\coprod_{\ell=1}^{\infty} \coprod_{\ell\left(\pi^{\times}(\xi)\right)=\ell}\left(c_{\mathrm{A}}^{(n)}\right)^{-1}\left(\xi ; \pi^{\times}(\xi)\right) \times \mu_{n}^{2} .
$$


Therefore $\left(c_{\mathrm{A}}^{(n)}\right)^{-1}(\xi) \times \mu_{n}^{2}$ is the countable disjoint union of the adelically locally closed sets

$$
X_{\xi ; \ell}:=\coprod_{\ell\left(\pi^{\times}(\xi)\right)=\ell}\left(c_{\mathbf{A}}^{(n)}\right)^{-1}\left(\xi ; \pi^{\times}(\xi)\right) \times \mu_{n}^{2} .
$$

Proof. Evidently we only have to check that each $X_{\xi ; \ell}$ is locally closed. But we have from Corollary 4.5 that each constituent set $\left(c_{\mathrm{A}}^{(n)}\right)^{-1}\left(\xi ; \pi^{\times}(\xi)\right) \times \mu_{n}^{2}$ is locally closed, so it just remains for us to observe that there are fewer than $n^{\ell}(<\infty)$ multiplicative partitions of $\xi$ of length $\ell$.

It follows from the preceding that in (4.8) the critical player $\left(\mathrm{SL}_{2}(k)_{\mathrm{A}} \times \mu_{n}\right)^{2}$ can be decomposed as

$$
\begin{aligned}
\left(\mathrm{SL}_{2}(k)_{\mathbf{A}} \times \mu_{n}\right)^{2} & =\coprod_{\ell=1}^{\infty} \coprod_{\xi \in \mu_{n}} \coprod_{\ell\left(\pi^{\times}(\xi)\right)=\ell}\left(c_{\mathbf{A}}^{(n)}\right)^{-1}\left(\xi ; \pi^{\times}(\xi)\right) \times \mu_{n}^{2} \\
& =\coprod_{\ell=1}^{\infty} \coprod_{\xi \in \mu_{n}} X_{\xi ; \ell} .
\end{aligned}
$$

So, finally, and in view of what will transpire in the next section, write $\mu=\mu_{n}, X_{0}=\mathrm{SL}_{2}(k)$, $X_{\mathbf{A}}=\mathrm{SL}_{2}(k)_{\mathrm{A}}$, and $\widetilde{X}_{\mathbf{A}}=\widetilde{\mathrm{SL}}_{2}(k)_{\mathrm{A}}^{(n)}$, for notational ease, and rewrite $(4.8)$ as

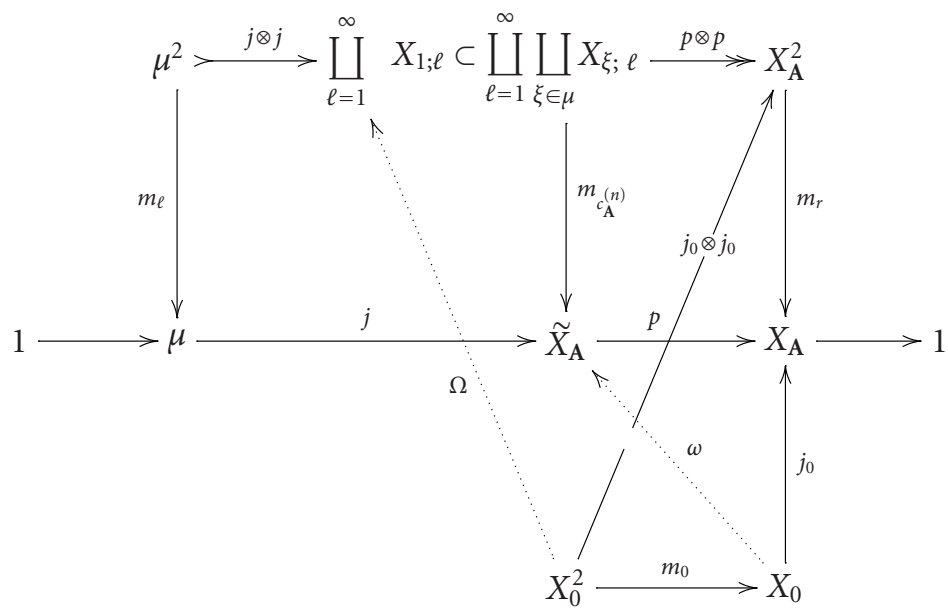

\section{Interpretations of splitting}

We noted in the preceding section, in connection with (4.8) and (4.9) and so also in connection with (4.20), that $p \circ \omega=j_{0}$ is equivalent to $(p \otimes p) \circ \Omega=j_{0} \otimes j_{0}$, staying with the abuse of notation in (4.9). This is trivial in light of (4.8) but it behooves us to observe that the formalism of (4.20) suggests that $\Omega$ should be of the form

$$
\begin{gathered}
\Omega=\left(\mathrm{id} \otimes s_{\mathbf{A}}\right) \otimes\left(\mathrm{id} \otimes s_{\mathbf{A}}\right): \mathrm{SL}_{2}(k)^{2} \longrightarrow\left(\mathrm{SL}_{2}(k)_{\mathbf{A}} \times \mu_{n}\right)^{2}, \\
\left(\sigma_{1}, \sigma_{2}\right) \longmapsto\left(\sigma_{1}, s_{\mathbf{A}}\left(\sigma_{1}\right), \sigma_{2}, s_{\mathbf{A}}\left(\sigma_{2}\right)\right) .
\end{gathered}
$$


We also noted that having $\omega$ split $c_{\mathrm{A}}^{(n)}$ on $\mathrm{SL}_{2}(k)$, or, equivalently, having $\omega$ split $\tilde{X}_{\mathbf{A}}$ on $X_{0}$ as indicated in (4.20), is just the stipulation that for all $\sigma_{1}, \sigma_{2} \in \mathrm{SL}_{2}(k)$

$$
s_{\mathbf{A}}\left(\sigma_{1}\right) s_{\mathbf{A}}\left(\sigma_{2}\right)=s_{\mathbf{A}}\left(\sigma_{1} \sigma_{2}\right) c_{\mathbf{A}}^{(n)}\left(\sigma_{1}, \sigma_{2}\right),
$$

and the strong form of splitting given by Kubota in [20] is simply the statement that $s_{\mathrm{A}}$ should be a group homomorphism. We will summarize these things in the form of a single central proposition, below, the proof of which is really already present in the earlier parts of this paper. It is important for our larger aims to fit this proposition into as spare a diagrammatical setting as possible, seeing that we seek to bring sheaf-theoretic methods into play. Therefore, the task facing us now is to cast (5.2) in the form of the commutativity of a diagram closely related to (4.8) and (4.20). Before long we will pass to a "dual" diagram where structure sheaves take the place of topological spaces so as to be in a position to address the existence of something of a dual morphism to $s_{\mathrm{A}}$ by means of derived categories. It is in this latter connection that Corollary 4.6 acquires its justification: the fact that the $X_{\xi ; \ell}$ are locally closed, so that $\bigsqcup_{\xi \in \mu} X_{\xi}$; is too, facilitates the definition of stratifications in the sense of [1].

First of all, if

$$
\begin{gathered}
j^{0}: \widetilde{\mathrm{SL}}_{2}(k)_{\mathrm{A}}^{(n)} \longrightarrow \mu_{n}, \\
(\sigma, \xi) \longmapsto \xi,
\end{gathered}
$$

or $j^{0}: \tilde{X}_{\mathbf{A}} \rightarrow \mu$ (in contrast with $\left.j:(\sigma, \xi) \mapsto \sigma\right)$, and, accordingly,

$$
\begin{gathered}
j^{0} \otimes j^{0}:\left(\mathrm{SL}_{2}(k)_{\mathbf{A}} \times \mu_{n}\right)^{2} \longrightarrow \mu_{n}^{2}, \\
\left(\sigma_{1}, \xi_{1}, \sigma_{2}, \xi_{2}\right) \longmapsto\left(\xi_{1}, \xi_{2}\right),
\end{gathered}
$$

then we quickly get the following amplification of the critical part of (4.20). However, this harbors a more-than-semantical difficulty in that $m_{c_{\mathrm{A}}^{(n)}}$ 's continuity is compromised because of possible discontinuities in $c_{\mathrm{A}}^{(n)}$ 's local factors (as per the example in Section 4):

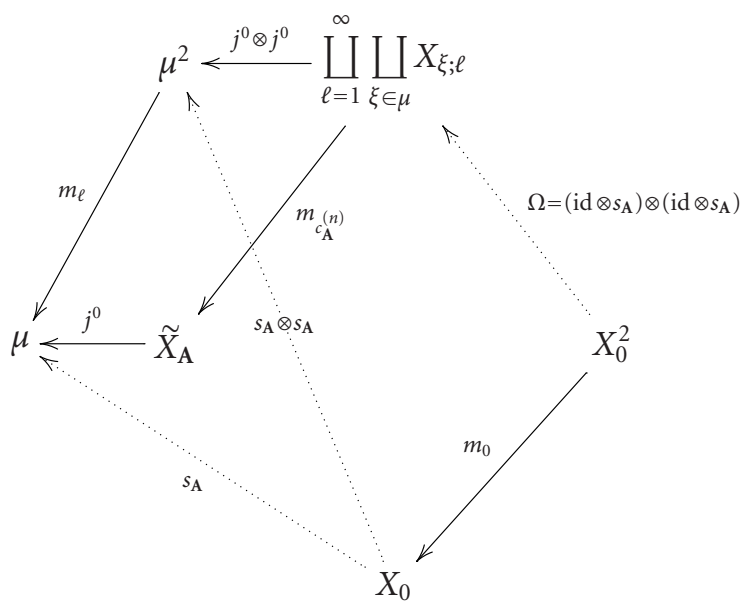


Now, although (5.5) is not properly a diagram in the category of topological spaces, we can extricate ourselves from this problem by invoking our earlier observation that in the equivalence classes $\left(c_{\mathrm{A}}^{(n)}\right)^{-1}(\xi) \times \mu_{n}^{2}=\coprod_{\ell=1}^{\infty} X_{\xi ; \ell}$, whose union over $\xi \in \mu_{n}$ evidently exhausts the target space for $\Omega$, the problematical action of $m_{c_{\mathrm{A}}^{(n)}}$ is just ordinary multiplication in $\mathrm{SL}_{2}(k)_{\mathbf{A}} \times \mu_{n}$ followed by translation in the second coordinate by $\xi$ (fixed). So, $m_{c_{\mathrm{A}}^{(n)}}$ obviously acts continuously on each $\coprod_{\ell=1}^{\infty} X_{\xi ; \ell}$. So, at the cost of adding yet another set of diagrams to the mix, we convey this as follows, with the meanings of $m_{\xi ; c_{A}^{(n)}}$ and $\otimes_{\xi \in \mu} m_{\xi ; c_{\mathrm{A}}^{(n)}}=\otimes_{\xi} m_{\xi ; c_{\mathrm{A}}^{(n)}}$ being the obvious ones:

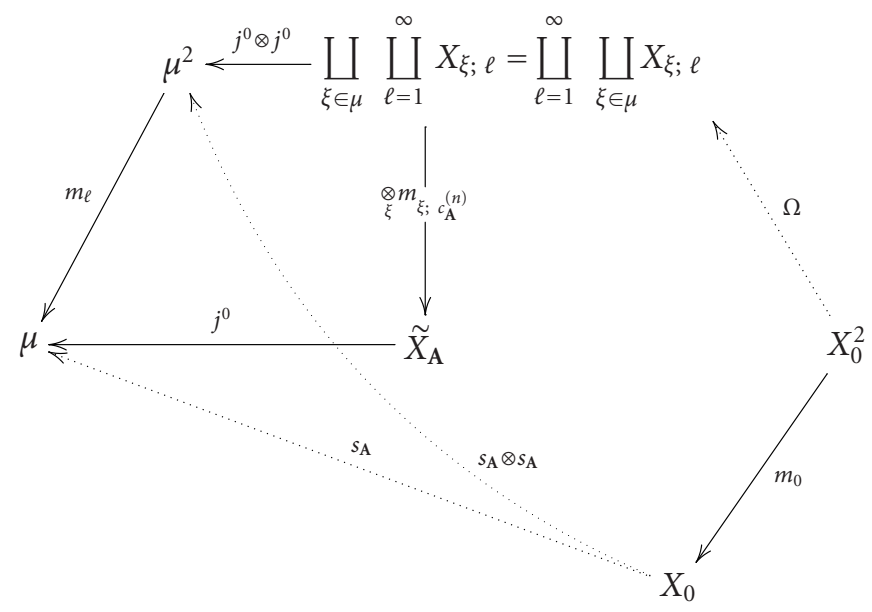

so that, for each $\xi_{0} \in \mu_{n}$,

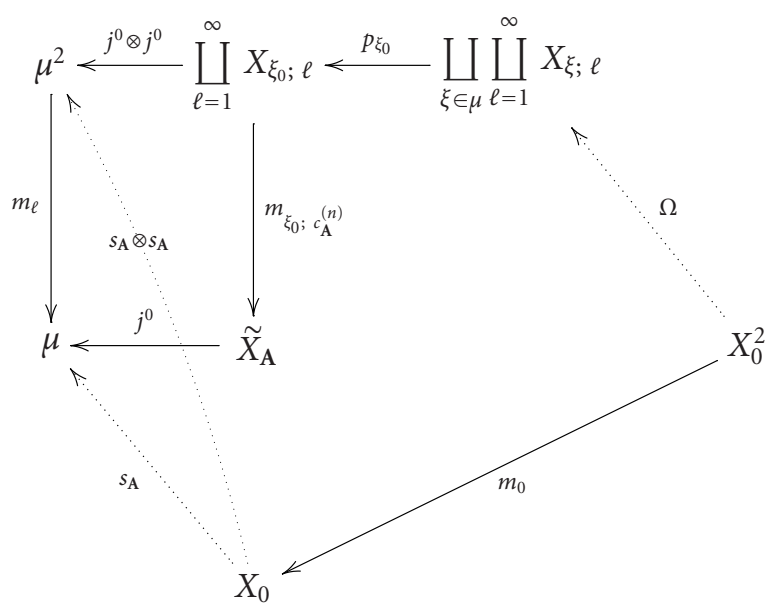


Admittedly there is still some apparent difficulty qua categorical membership due to the evident discontinuity of the projection $p_{\xi_{0}}$. However, this can be circumvented by going after not $\Omega$ but, first, $\Omega_{\xi_{0}}$ as given in (5.8) below. Under these circumstances the pathology of the $p_{\xi_{0}}$ disappears from the game and, subsequently, the collective data $\left\{\Omega_{\xi_{0}}\right\}_{\xi_{0} \in \mu}$ suffices to give us $\Omega$ :

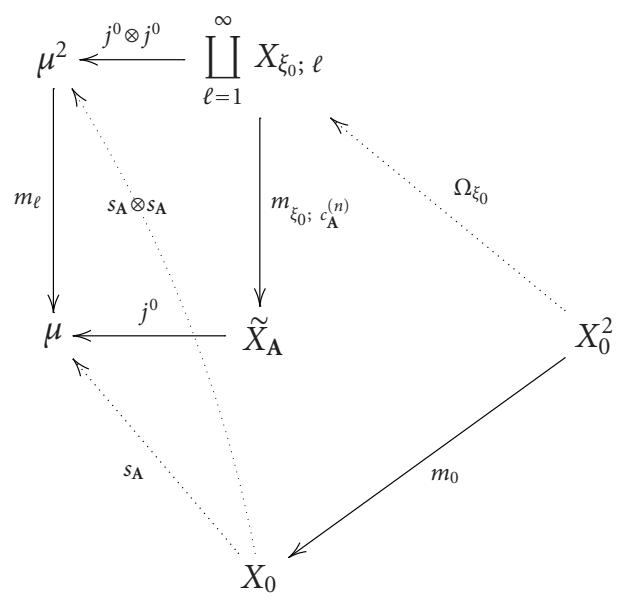

Each object in (5.8) is a topological space and each mapping (or arrow) in (5.8) is continuous, which is to say a morphism.

And now we come to the aforementioned central proposition.

Proposition 5.1. (i) In (4.20), (5.5), (5.6), (5.7), and (5.8) the existence of $s_{\mathrm{A}}$, that of $s_{\mathrm{A}} \otimes s_{\mathrm{A}}$, and that of $\Omega$ (as given in (5.1)) are all equivalent.

(ii) The mappings $s_{\mathrm{A}}, s_{\mathrm{A}} \otimes s_{\mathrm{A}}$ are group homomorphisms if and only if $\left.c_{\mathrm{A}}^{(n)}\right|_{X_{0}^{2}} \equiv 1$, which is to say (cf. (4.6), (4.17), and (4.18)) that $\mathrm{im}(\Omega) \subset \coprod_{\ell=1}^{\infty} X_{1 ; \ell}$.

(iii) The (group homo-) morphism $\omega=\mathrm{id} \otimes s_{\mathrm{A}}$ splits $\tilde{X}_{\mathrm{A}}$ on $X_{0}$ if and only if the following subdiagram of (5.8) is commutative, in the category of topological spaces, for every $\xi_{0} \in \mu$ :

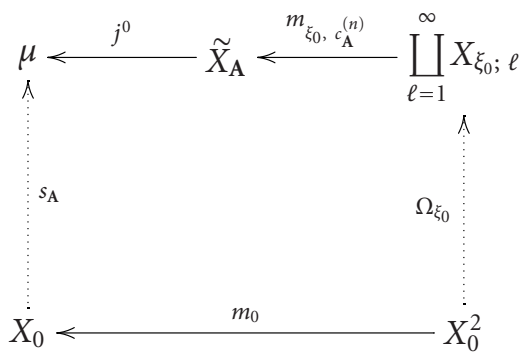


(iv) Suppose that in place of $\Omega=\left(\mathrm{id} \otimes s_{\mathrm{A}}\right) \otimes\left(\mathrm{id} \otimes s_{\mathrm{A}}\right)$ (as per (5.1) in (5.6), (5.7), (5.8)) we have merely a morphism, say,

$$
\Xi: X_{0}^{2} \longrightarrow\left(X_{\mathbf{A}} \times \mu\right)^{2}=\coprod_{\ell=1}^{\infty} \coprod_{\xi \in \mu} X_{\xi ; \ell},
$$

(cf. (4.5)) such that

$$
\operatorname{im}(\Xi) \subset \coprod_{\ell=1}^{\infty} X_{1 ; \ell}
$$

(cf. (4.6)). This is sufficient (and necessary) for $n$-Hilbert reciprocity.

Proof. (i) The existence of $s_{\mathrm{A}}$ is obviously equivalent to that of $s_{\mathrm{A}} \otimes s_{\mathrm{A}}$. Beyond this the equivalence of the existence of $s_{\mathrm{A}}$ and that of $\Omega=\left(\mathrm{id} \otimes s_{\mathrm{A}}\right) \otimes\left(\mathrm{id} \otimes s_{\mathrm{A}}\right)$ follows from the obvious equivalence of $p \circ \omega=j_{0}$ and $(p \otimes p) \circ \Omega=j_{0} \otimes j_{0}$.

(ii) This follows from (5.2).

(iii) The commutativity of (5.9), for all $\xi_{0} \in \mu$, is precisely the commutativity of

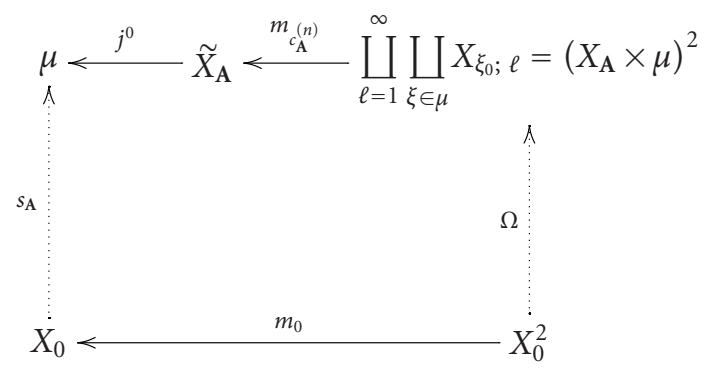

Now the commutativity of $(5.12)$ is precisely the statement that $s_{\mathrm{A}} \circ m_{0}=j_{0} \circ m_{c_{\mathrm{A}}^{(n)}} \circ \Omega$. Applying the left expression to $\left(\sigma_{1}, \sigma_{2}\right) \in X_{0}^{2}=\mathrm{SL}_{2}(k)^{2}$ produces the left side of $(5.2)$, while applying the right expression to $\left(\sigma_{1}, \sigma_{2}\right)$ produces the right side of $(5.2)$.

(iv) This is immediate from our discussion at the end of Section 3.

The thrust of Proposition 5.1 is that with (5.9) situated in the category of topological spaces we are at last poised for an implementation of the methodology of derived categories in order to address Hecke's challenge in a new way.

\section{The abelian category of sheaves over $\tilde{X}_{\mathrm{A}}$}

With (5.9) being a diagram in the category of topological spaces we now set out to devise a diagram of sheaves on the appropriate site, that is, the appropriate vertex of (5.9), geared toward carrying the arithmetical information of $n$-Hilbert reciprocity. In other words, we seek to recast the indicated parts of Proposition 5.1 in terms of the homological 
algebra that pertains first to sheaves, then to derived categories of sheaf complexes, and, subsequently, we will have to stipulate suitable properties of the various sheaves that arise so as to capture all the relevant arithmetical structure. As this makes for a natural twopart strategy, we take the liberty of developing the first part, involving as yet unspecified sheaves and morphisms, in general terms, leaving the various choices till later. However, we add a few inter alia remarks in anticipation of these assignments and choices. We summarize things in Section 8 (and then proceed to look ahead).

Since $\widetilde{X}_{\mathrm{A}}$ carries a copy of $X_{0}=\mathrm{SL}_{2}(k)$ and a copy of $\mu=\mu_{n}$ within itself, it is natural to assign it the role of base space (or site, in the sense of Grothendieck), supporting an array of sheaves designed to convey $n$-Hilbert reciprocity. Write ? for ${ }^{*}$ or ! as per Grothendieck's notation for his "six functors" (and then some); we also reserve the right to replace, for instance, a sheaf morphism $f^{!}$by $R f^{!}$, where $R$ denotes Verdier's derived functor, should the need arise.

So, if $X \stackrel{f}{\rightarrow} Y$ is a continuous mapping of topological spaces, we get, covariantly, $f_{*}$ and $f_{!}$mapping sheaves on $X$ to sheaves on $Y$, and, contravariantly, $f^{*}$ and $f^{!}$mapping the other way. Of course, $f_{*}$ (resp., $f^{*}$ ) is just the direct (resp., inverse) image functor attached to $f$, and then $f_{!}$and $f^{!}$are characterized by well-known adjointness properties relative to $f_{*}$ and $f^{*}$. For further details, see $[5,6,7,16]$.

We begin by incorporating the morphism

$$
\begin{aligned}
i \otimes 1: \mathrm{SL}_{2}(k)=X_{0} & \longrightarrow \mathrm{SL}_{2}(k)_{\mathrm{A}} \times_{c_{\mathrm{A}}^{(n)}} \mu_{n}=\tilde{X}_{\mathrm{A}}, \\
\sigma & \longmapsto(\sigma, 1)
\end{aligned}
$$

into (5.9) (redrawn as per (5.8)):

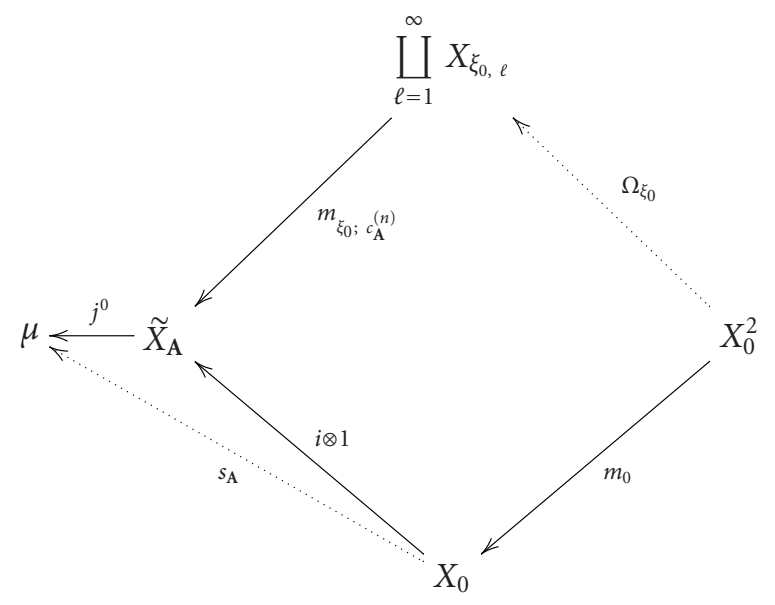


With $\mathscr{F}$ an a priori unspecified sheaf on $\tilde{X}_{\mathrm{A}}$, identified with its sheaf space [25] or espace étalé, we get the following sheaf diagrams relative to the vertices of (6.2):
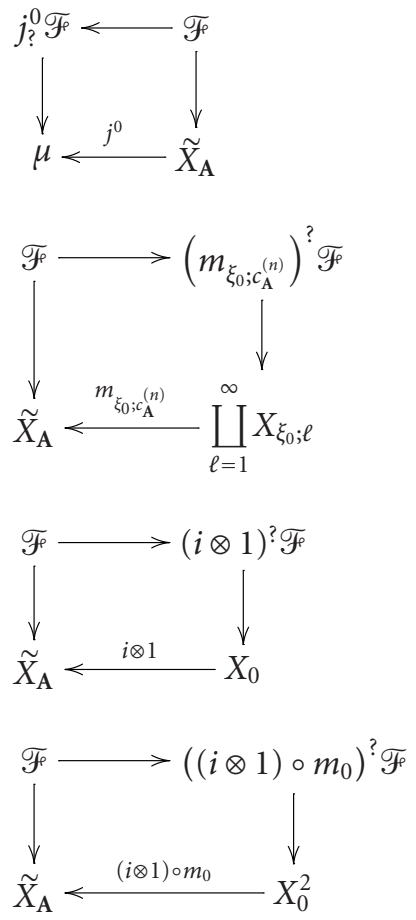

Thus, taking note of the fact that, in general, with the ? varying individually, $f$ ? $f^{\text {? }}$ and $\left[f^{?} f_{?}\right]$ do not cancel out to identity morphisms, we get, as sheaves on $\tilde{X}_{\mathbf{A}}$,
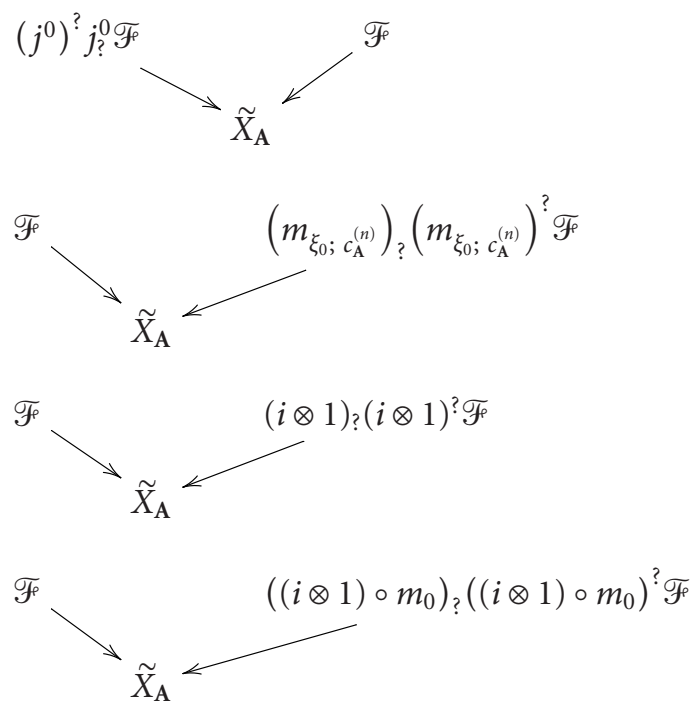
As we suggested above, a critical future task is to specify properties imposed on $\mathscr{F}$ (or on a collection of $\mathscr{F}$ 's) consonant with the action of $c_{\mathrm{A}}^{(n)}$, that is, with $n$-Hilbert reciprocity. A large part of this specification is the definition of maps $\iota, v, \iota^{0}, \nu^{0}$ as follows:

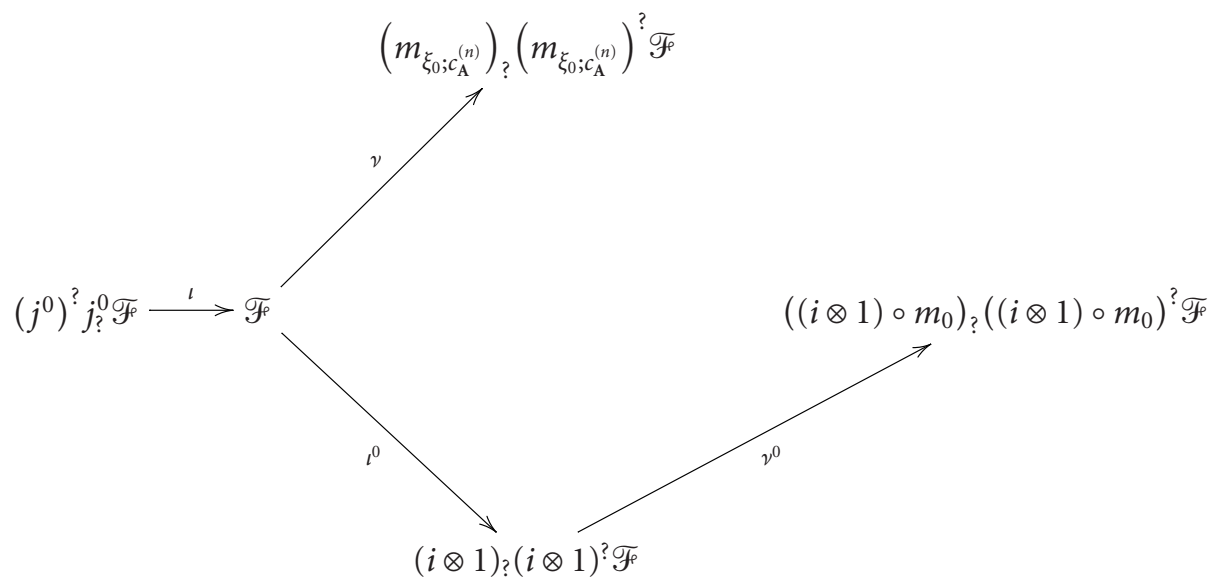

(This is an incomplete diagram in the category of sheaves on $\tilde{X}_{\mathrm{A}}$.) In order to simplify notation, write $\left(j^{0}\right)^{?} j_{?}^{0} \mathscr{F}=\mathscr{A},\left(m_{\xi_{0} ; c_{\mathrm{A}}^{(n)}}\right)_{?}\left(m_{\xi_{0} ; c_{\mathrm{A}}^{(n)}}\right)^{?} \mathscr{F}=\mathscr{B},\left((i \otimes 1) \circ m_{0}\right)_{?}\left((i \otimes 1) \circ m_{0}\right)^{?} \mathscr{F}=$ $\mathscr{C}$ and $(i \otimes 1)_{?}(i \otimes 1)^{?} \mathscr{F}=\mathscr{D}$, and get

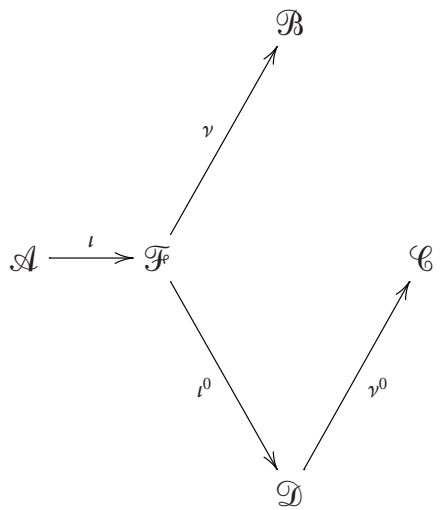

Suppose, now, that we have, say, $\Phi=\Phi(\mathscr{F}) \in \operatorname{Hom}(\mathscr{B}, \mathscr{C})$ in hand. Then the next (and essentially final) step is to infer from the existence of $\Phi(\mathscr{F})$ the unique determination of 
$\Omega_{\xi_{0}}$ as situated thus:

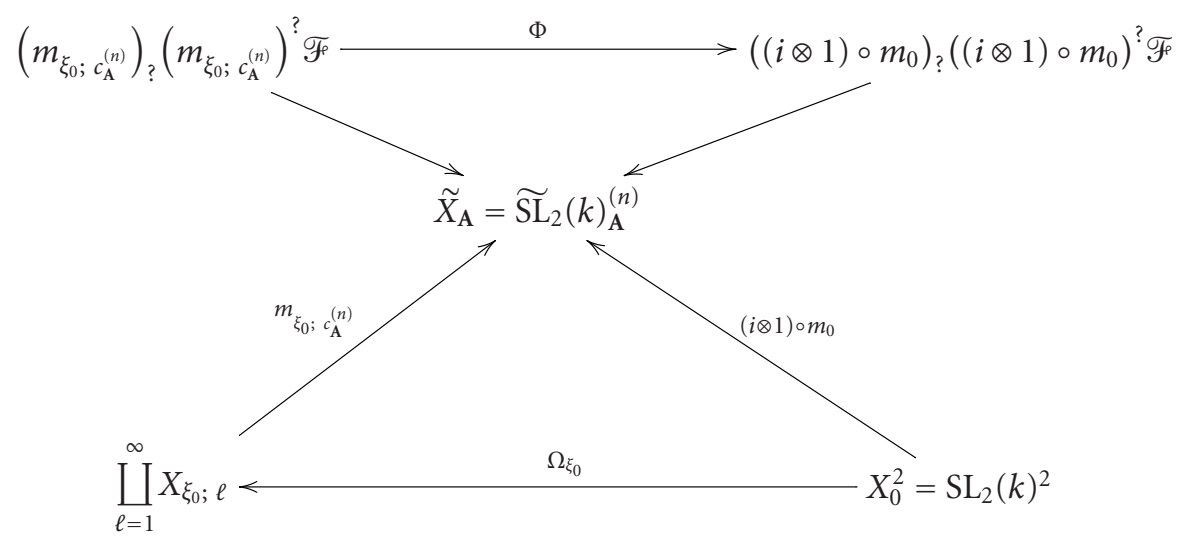

Therefore, the idea is that the existence of (possibly sufficiently many) $\Phi(\mathscr{F})$ as per

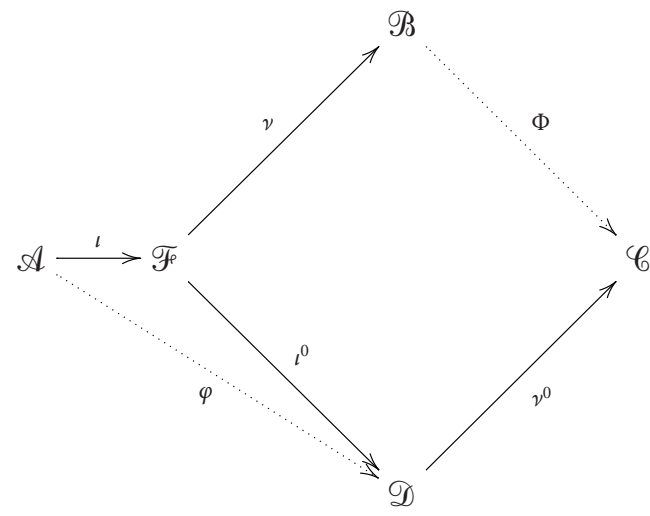

for suitably characterized $\mathscr{F}$, is enough to yield the critical existence of $\Omega_{\xi_{0}}$ as in (6.2). Proposition 5.1 then actually yields that getting $\Omega_{\xi_{0}}$ for all $\xi_{0} \in \mu_{n}$ is enough as far as $n$ Hilbert reciprocity goes. However, we have included $\varphi$, in (6.8) parallel to $s_{\mathrm{A}}$ just as $\Phi$ is parallel to $\Omega_{\xi_{0}}$, in order to cover the full structure of (5.8). Naturally a diagram similar to (6.7) holds with $\varphi$ (resp., $s_{\mathrm{A}}$ ) in place of $\Phi$ (resp., $\Omega_{\xi_{0}}$ ).

Beyond this the preceding developments are also based on the choice of $i \otimes 1$, as given by (6.1), to imbed $\mathrm{SL}_{2}(k)=X_{0}$ in $\widetilde{S L}_{2}(k)_{\mathrm{A}}^{(n)}=\widetilde{X}_{\mathrm{A}}$. Obviously there are other options available, for example, of the form $i \otimes \xi$, where $\xi \in \mu_{n}$ abusively signifies the indicated constant map.

In any event, with the precise formulation of the connection between $\Phi$ and $\Omega_{\xi_{0}}$ left for the future, we now turn to diagram (6.8) proper. In a (still) somewhat vague fashion, we may regard it as "dual" to (5.9), and the first order of business is to get at $\Phi$ 's existence as a question of homological algebra in the derived category defined on the abelian category of sheaves on $\tilde{X}_{\mathrm{A}}$. 


\section{The derived category of sheaf complexes on $\tilde{X}_{\mathrm{A}}$}

First here are some generalities (consult $[12,16]$ ). If $\mathfrak{A}$ is an abelian category, its associated derived category $D(\mathfrak{A})$ is formed by localizing the category of chain complexes from $\mathfrak{A}$, taken modulo chain homotopy, at the ideal of quasi-isomorphisms. (Two chain complexes are quasi-isomorphic if and only if they possess the same cohomology groups.) Such a derived category is in fact a triangulated category, meaning that if $A^{\bullet} \stackrel{f}{\longrightarrow} B^{\bullet}$ is any morphism of complexes, understood rightly, then the mapping cone construction $A^{\bullet} \stackrel{f}{\longrightarrow} B^{\bullet} \rightarrow Z_{f}^{\bullet}$ provides a notion of distinguished triangle subject (by definition) to a quartette of characterizing axioms. Essentially, we have that, with the proper sign conventions in place (as regards chain morphisms), rotations of distinguished triangles are again distinguished, as are isomorphs of distinguished triangles, and we also have that, for morphisms (ladders) between distinguished triangles, any two (rungs) determine the third.

It is a routine exercise to prove that any distinguished triangle $A^{\bullet} \stackrel{f}{\longrightarrow} B^{\bullet} \rightarrow Z^{\bullet}$ gives rise, mechanically, to two so-called long exact Hom-sequences. For our purposes the one of interest is of the form

$$
\begin{gathered}
\cdots \longleftarrow \operatorname{Hom}_{D(\mathfrak{A})}\left(Z^{\bullet}[-1], C^{\bullet}\right) \longleftarrow \operatorname{Hom}_{D(\mathfrak{A})}\left(A^{\bullet}, C^{\bullet}\right) \stackrel{f^{*}}{\longleftarrow} \operatorname{Hom}_{D(\mathfrak{A})}\left(B^{\bullet}, C^{\bullet}\right) \longleftarrow \\
\longleftarrow \operatorname{Hom}_{D(\mathfrak{A})}\left(Z^{\bullet}, C^{\bullet}\right) \longleftarrow \cdots
\end{gathered}
$$

Here $Z \bullet[-1]$ is the chain complex obtained by shifting $Z \bullet$ "one vertex to the right" (and abiding by a differential sign convention). By definition, a triangulated category comes equipped with a translation functor [1]: $X \mapsto X[1]$ (for any object $X$ ), automatically admitting iteration through all of $\mathbf{Z}$.

With $\mathfrak{A}$ the category of sheaves on $\tilde{X}_{\mathrm{A}}$, that is, $\mathfrak{A}=\mathfrak{S h} / \tilde{X}_{\mathrm{A}}$, just write $\mathfrak{D}$ in place of $D(\mathfrak{A})$. Associating a sheaf, for example, $\mathscr{F}_{\mathfrak{A}}$, with the complex $\mathscr{F}^{\bullet}$ which has each nonzero vertex equal to 0 and the 0 th vertex equal to $\mathscr{F}$ yields a natural imbedding of $\mathfrak{S h} / \tilde{X}_{\mathbf{A}}$ in D. Accordingly (6.8) evolves into

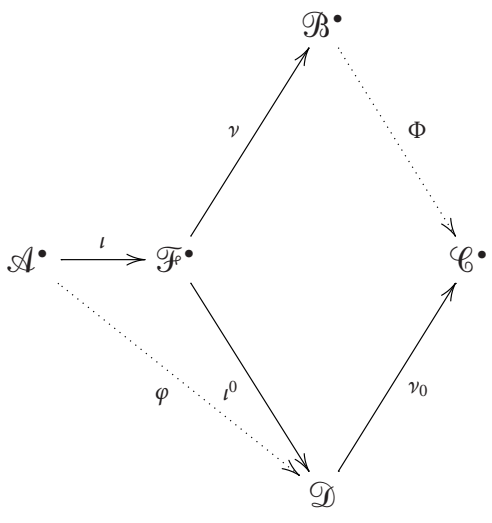

We now get the following proposition. 
Proposition 7.1. The existence of $\Phi$ follows if $\operatorname{Hom}_{\mathfrak{D}}\left(Z^{\bullet}[-1], \mathscr{C}^{\bullet}\right)=0$, where the morphism $\mathscr{F}^{\bullet} \stackrel{\nu}{\longrightarrow} \mathscr{B}^{\bullet}$ sits in some distinguished triangle $\mathscr{F}^{\bullet} \stackrel{\nu}{\longrightarrow} \mathscr{B}^{\bullet} \rightarrow Z \bullet$.

Proof. We get, from (7.1), the exactness of the long Hom-sequence

$$
\begin{gathered}
\cdots \longleftarrow \operatorname{Hom}_{\mathfrak{D}}\left(Z^{\bullet}[-1], \mathscr{C}^{\bullet}\right) \longleftarrow \operatorname{Hom}_{\mathfrak{D}}\left(\mathscr{F}^{\bullet}, \mathscr{C}^{\bullet}\right) \stackrel{v^{*}}{\longleftarrow} \operatorname{Hom}_{\mathfrak{D}}\left(\mathscr{B}^{\bullet}, \mathscr{C}^{\bullet}\right) \longleftarrow \\
\longleftarrow \operatorname{Hom}_{\mathfrak{D}}\left(Z^{\bullet}, \mathscr{C}^{\bullet}\right) \longleftarrow \cdots
\end{gathered}
$$

As always, $\nu^{*}$ is just the mapping taking $\mathscr{B} \bullet \rightarrow \mathscr{C}^{\bullet}$ to $\mathscr{F}^{\bullet} \rightarrow \mathscr{C}^{\bullet}$ factoring through $\nu$ (as well as $\left.\mathscr{B}^{\bullet} \rightarrow \mathscr{C}^{\bullet}\right)$. Therefore, if $\operatorname{Hom}_{\mathfrak{D}}\left(Z^{\bullet}[-1], \mathscr{C}^{\bullet}\right)=0$, as given, then $v^{*}$ is surjective and this implies that the morphism $\mathscr{F}^{\bullet} \stackrel{\nu^{0} \circ \iota^{0}}{\longrightarrow} \mathscr{C}^{\bullet}\left(\right.$ in $\operatorname{Hom}_{\mathfrak{D}}\left(\mathscr{F}^{\bullet}, \mathscr{C}^{\bullet}\right)$ ) possesses a preimage $\Phi \in \operatorname{Hom}_{\mathfrak{D}}\left(\mathscr{B} \bullet, \mathscr{C}^{\bullet}\right)$. In other words, $\nu^{*}(\Phi)=\Phi \circ \nu=\nu^{0} \circ \iota^{0}$ :

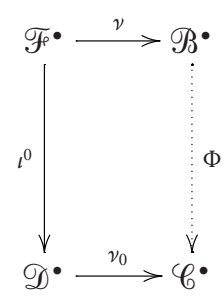

Thus, returning to our original notation, in order to obtain $\Phi=\Phi(\mathscr{F})$ as situated in the diagram (6.7), it suffices to have, in $\mathfrak{D}=D\left(\mathfrak{S h} / \tilde{X}_{\mathbf{A}}\right)$,

$$
\operatorname{Hom}_{\mathfrak{D}}\left(Z \cdot[-1],\left((i \otimes 1) \circ m_{0}\right)_{?}\left((i \otimes 1) \circ m_{0}\right)^{?} \mathscr{F}^{\bullet}\right)=0,
$$

where $Z \bullet$ completes a distinguished triangle based on

$$
v: \mathscr{F}^{\bullet} \longrightarrow\left(m_{\xi_{0} ; c_{\mathrm{A}}^{(n)}}\right)_{?}\left(m_{\xi_{0} ; c_{\mathrm{A}}^{(n)}}\right)^{?} \mathscr{F}^{\bullet}
$$

(as in (6.5)) and $\nu$ is a morphism to be defined, as is the case for $\mathscr{F}_{\mathscr{F}}$ (and $\mathscr{F}^{\bullet}$ ). We note that (7.5) is a very demanding condition in view of the fact that a morphism of chain complexes in $\mathfrak{D}$ is represented by 0 if and only if it composes with some quasi-isomorphism to yield a mapping which is chain homotopic to 0 (see [12, pages 38-39]). Beyond this we face the task of deciding the proper (possibly different) substitutions for the two ?'s which occur, of course, and a good deal more besides; see the next section.

\section{A preview of future work}

Taking stock of what we have done in the preceding pages and looking toward what lies ahead, we can discern a pair of interconnected themes. One of these concerns the top row of (4.20), regarded as a sequence of continuous mappings between topological spaces, foremost among them is the inclusion $\coprod_{\ell=1}^{\infty} X_{1 ; \ell} \subset \coprod_{\ell=1}^{\infty} \coprod_{\xi \in \mu} X_{\xi ; \ell}$. The sets $X_{\xi ; \ell}$, as given by (4.18), are locally closed and the tactical reasons for our identification of sets of such a special nature are twofold. In the first place, getting $\Omega$ to map entirely into $\coprod_{\ell=1}^{\infty} X_{1 ; \ell}$ would produce $n$-Hilbert reciprocity immediately, following Proposition 5.1(iv). In the 
second place (and a more reasonable prospect), getting $\Omega$ to map into the larger set $\bigsqcup_{\ell=1}^{\infty} \bigsqcup_{\xi \in \mu} X_{\xi ; \ell}$ (cf. (5.10) as well as the remarks below) might be facilitated by introducing suitable perversities on this target site once we have effected the indicated transition to our proposed dual setting of sheaves and sheaf complexes. In order to introduce the machinery of perverse sheaves, locally closed strata are of course indicated $[1,16]$.

The other theme concerns the diagrams (5.8) and (6.2), both of which spring forth from (4.20). We see from Proposition 5.1(i), (ii), (iii) that the critical issue is to prove the existence of $\Omega$ (as already suggested in the preceding), that is, of the $\Omega_{\xi_{0}}$ for all $\xi_{0} \in \mu$. We propose to go at this by means of exploiting the homological algebra pertaining to the derived category $\mathfrak{D}=D\left(\mathfrak{S h} / \tilde{X}_{\mathbf{A}}\right)$. By Proposition 5.1 (iii) the existence (and shape) of $\Omega$ should be the final word on Hecke's challenge; going to the quasi-dual diagram (6.5) we see that getting at $\Phi$ 's existence, as something of an avatar of $\Omega_{\xi_{0}}$, is a "homological" affair. In other words, by Proposition 7.1 we find that everything comes down to determining suitable $\mathscr{F}_{\mathscr{F}}$ such that $\operatorname{Hom}_{\mathfrak{D}}\left(Z \bullet[-1],\left((i \otimes 1) \circ m_{0}\right)_{?}\left((i \otimes 1) \circ m_{0}\right)^{\text {? }} \mathscr{F}_{\mathscr{F}} \bullet\right)=0$, where $\mathscr{F}_{\bullet}$ can be situated in some appropriate distinguished triangle of the form $\mathscr{F}_{\bullet} \stackrel{\nu}{\rightarrow}$ $\left(m_{\xi_{0} ; c_{\mathrm{A}}^{(n)}}\right) ?\left(m_{\xi_{0} ; c_{\mathrm{A}}^{(n)}}\right)^{?} \mathscr{F}_{\mathscr{f}}^{\bullet} \rightarrow Z \bullet$.

Thus center stage is soon assumed by the question of the arithmetical nature of $\mathscr{F}_{F}$ as (at first) a sheaf on $\tilde{X}_{\mathrm{A}}$. This means that $\mathscr{F}$ must be designed not only so as to yield (7.5) but also to provide the completion of the hugely important diagram (6.7): $\mathscr{F}_{f}$ should be chosen such that the existence of $\Phi=\Phi(\mathscr{F})$, or of a class of such morphisms in $\mathfrak{D}$, precipitates the existence of $\Omega_{\xi_{0}}$. Then varying $\xi_{0}$ across $\mu=\mu_{n}$ will yield $\Omega$. The determination of $\mathscr{F}_{F}$ is a multifaceted affair, involving the imperative of properly covering the action of $c_{\mathbf{A}}^{(n)} \in H^{2}\left(\mathrm{SL}_{2}(k)_{\mathrm{A}}, \mu_{n}\right)$, the half dozen or so individual assignments of * or ! to ? (not to mention what Verdier's $R$ might bring about when we go to the derived category), and the stipulation of $\iota, v, \iota^{0}, v^{0}$. And $\mathscr{F}$ must also be rigged so as to provide the vanishing of the above Hom group as per Proposition 7.1. This is a relatively subtle business, only one major aspect of which is the matter of choosing the right distinguished triangle in which to fit $\nu$. We are quickly faced with rather intricate technical questions concerning the indicated derived category as already mentioned at the end of Section 7 . There is a real likelihood that our choice of (6.1) as the requisite imbedding of the rational points in our base site $\tilde{X}_{\mathbf{A}}$ will have to be revisited: the morphisms $i \otimes \xi_{0}$, with $\xi_{0} \in \mu$, are obvious candidates.

In any event, the next phase of the campaign will be concerned with the behavior of our (quasi-) dual formalism of sheaves on $\widetilde{X}_{\mathrm{A}}=\widetilde{\mathrm{SL}}_{2}(k)_{\mathrm{A}}^{(n)}$ instead of Kubota's original formalism of the metaplectic group $\widetilde{\mathrm{SL}}_{2}(k)_{\mathrm{A}}^{(n)}$ itself. We have presented, in the present article, the scaffolding for this enterprise whose ultimate goal is the resolution of Hecke's challenge to provide the analytic proof of general reciprocity for a number field. As we stress throughout [4], Hecke's own treatment of the quadratic case in [15], as well as Weil's in [26], should be classed, properly speaking, as Fourier analytic; indeed, both Hecke and Weil gain victory by a masterful exploitation of Fourier transform tactics culminating in $\vartheta$-functional equations. Following Grothendieck, functional equations should be encouraged to evolve to theorems about, say, Poincaré duality or Serre duality. In our proposed context of the derived category of sheaf complexes on $\tilde{X}_{\mathrm{A}}$, possibly with perversities to be defined before too long, we should look toward duality theorems per sé, that is, special 
correspondences, doubtless in connection with (6.7) and the relationship between $\Phi$ and $\Omega_{\xi_{0}}$. Recent work in the area of microlocal analysis (see $[16,22]$, e.g.) has centered on using the Fourier-Sato transform in a very suggestive manner.

\section{References}

[1] A. A. Bẹlinson, J. Bernstein, and P. Deligne, Faisceaux pervers [Perverse sheaves], Analysis and Topology on Singular Spaces, I (Luminy, 1981), Astérisque, vol. 100, Société Mathématique de France, Paris, 1982, pp. 5-171 (French).

[2] M. Berg, On a generalization of Hecke 9-functions and the analytic proof of higher reciprocity laws, J. Number Theory 44 (1993), no. 1, 66-83.

[3] - On local objects attached to 9-and $\zeta$-functions, Integral Transforms Spec. Funct. 10 (2000), no. 1, 13-24.

[4] The Fourier-Analytic Proof of Quadratic Reciprocity, Pure and Applied Mathematics, Wiley-Interscience, New York, 2000.

[5] P. Deligne, La conjecture de Weil. I, Inst. Hautes Études Sci. Publ. Math. (1974), no. 43, 273-307 (French).

[6] L L L L conjecture de Weil. II, Inst. Hautes Études Sci. Publ. Math. (1980), no. 52, 137-252 (French).

[7] P. Deligne (ed.), Séminaire de Géométrie Algébrique du Bois-Marie 4 $\frac{1}{2}$, Springer-Verlag, Berlin, 1977.

[8] H. M. Edwards, Riemann's zeta function, Pure and Applied Mathematics, vol. 58, Academic Press, New York, 1974, (Appendix).

[9] P. B. Garrett, Holomorphic Hilbert Modular Forms, The Wadsworth \& Brooks/Cole Mathematics Series, Wadsworth \& Brooks/Cole Advanced Books \& Software, California, 1990.

[10] S. S. Gelbart, Weil's Representation and the Spectrum of the Metaplectic Group, Lecture Notes in Mathematics, vol. 530, Springer-Verlag, Berlin, 1976.

[11] S. S. Gelbart and I. Piatetski-Shapiro, On Shimura's correspondence for modular forms of halfintegral weight, Automorphic Forms, Representation Theory and Arithmetic (Bombay, 1979), Tata Inst. Fund. Res. Studies in Math., vol. 10, Tata Institute of Fundamental Research, Bombay, 1981, pp. 1-39.

[12] R. Hartshorne, Residues and Duality, Lecture Notes in Mathematics, no. 20, Springer-Verlag, Berlin, 1966.

[13] E. Hecke, Über die Bestimmung Dirichletscher Reihen durch ihre Funktionalgleichung, Math. Ann. 112 (1936), 664-699 (German).

[14] _ Mathematische Werke, Vandenhoeck \& Ruprecht, Göttingen, 1970, article 33.

[15] _ Vorlesungen über die Theorie der algebraischen Zahlen, Chelsea Publishing, New York, 1970, 2nd ed. of the 1923 original, with an index.

[16] M. Kashiwara and P. Schapira, Sheaves on Manifolds, Springer-Verlag, Berlin, 1980, 2002.

[17] D. A. Kazhdan and S. J. Patterson, Metaplectic forms, Inst. Hautes Études Sci. Publ. Math. 59 (1984), 35-142.

[18] H. Koch, Algebraic Number Theory, Springer-Verlag, Berlin, 1997.

[19] T. Kubota, Topological covering of SL(2) over a local field, J. Math. Soc. Japan 19 (1967), no. 1, $114-121$.

[20] - On Automorphic Functions and the Reciprocity Law in a Number Field, Lectures in Mathematics, Department of Mathematics, Kyoto University, no. 2, Kinokuniya BookStore, Tokyo, 1969.

[21] H. Matsumoto, Sur les sous-groupes arithmétiques des groupes semi-simples déployés, Ann. Sci. École Norm. Sup. (4) 2 (1969), 1-62 (French). 


\section{Derived categories and general reciprocity}

[22] A. Polishchuk, Abelian Varieties, Theta Functions and the Fourier Transform, Cambridge Tracts in Mathematics, vol. 153, Cambridge University Press, Cambridge, 2003.

[23] B. Riemann, Über die Anzahl der Primzahlen unter einer gegebenen Grösse, Monatsber. Königl. Akad. Wiss. Berlin (1859), 671-680.

[24] J. Tate, Fourier analysis in number fields and Hecke's Zeta-functions, Ph.D. thesis, Princeton University, New Jersey, 1950, In Algebraic Number Theory, Academic Press, Sussex, 1967.

[25] B. R. Tennison, Sheaf Theory, London Mathematical Society Lecture Note Series, no. 20, Cambridge University Press, Cambridge, 1975.

[26] A. Weil, Sur certains groupes d'opérateurs unitaires, Acta Math. 111 (1964), 143-211 (French).

[27] - Remarks on Hecke's lemma and its use, Algebraic Number Theory (Kyoto Internat. Sympos., Res. Inst. Math. Sci., Univ. Kyoto, Kyoto, 1976), Japan Society for the Promotion of Science, Tokyo, 1977, pp. 267-274.

Michael Berg: Department of Mathematics, Loyola Marymount University, Los Angeles, CA 90045, USA

E-mail address: mberg@lmu.edu 


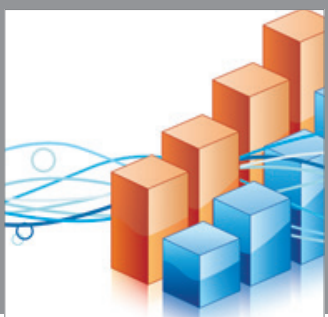

Advances in

Operations Research

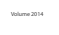

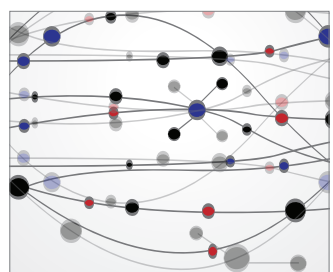

\section{The Scientific} World Journal
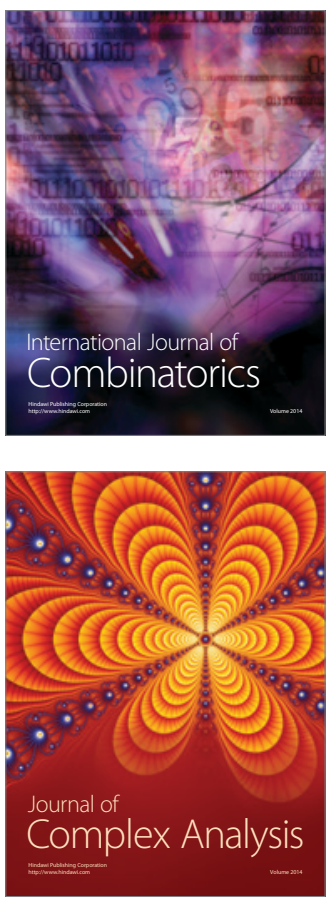

International Journal of

Mathematics and

Mathematical

Sciences
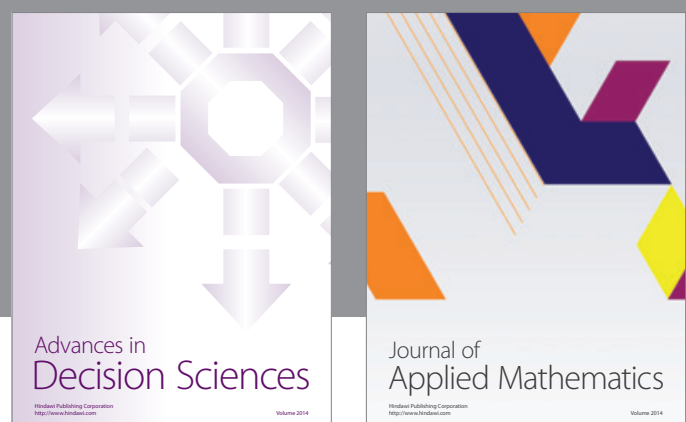

Journal of

Applied Mathematics
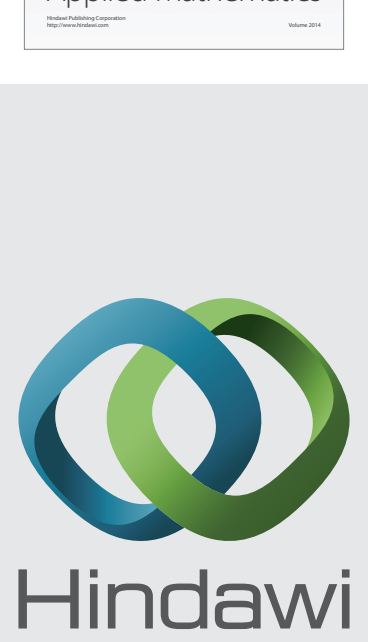

Submit your manuscripts at http://www.hindawi.com
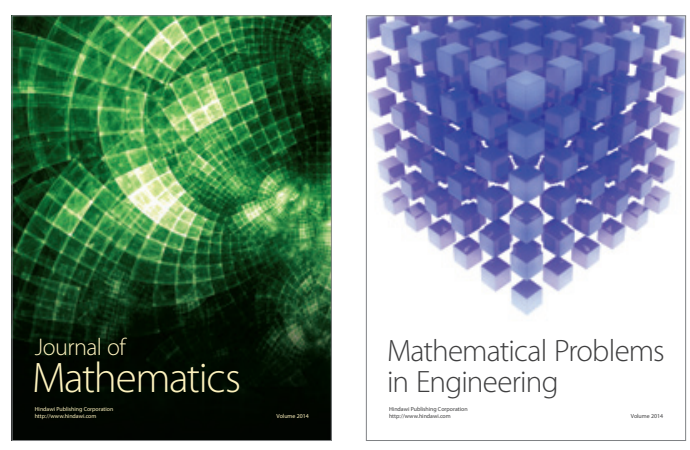

Mathematical Problems in Engineering
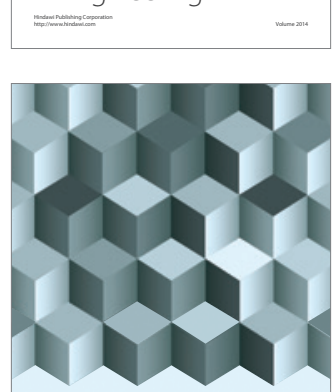

Journal of

Function Spaces
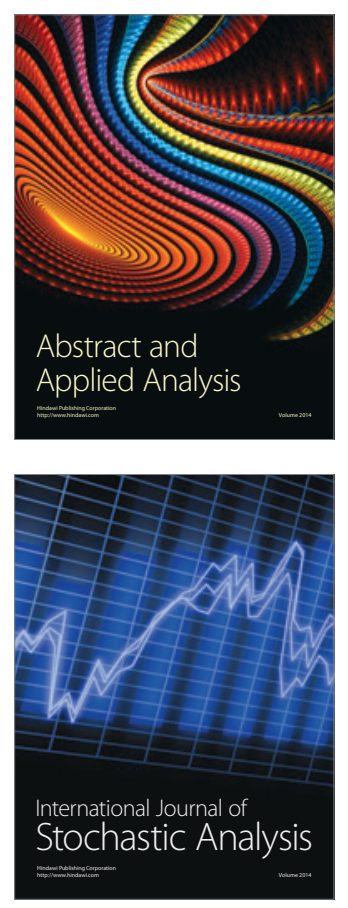

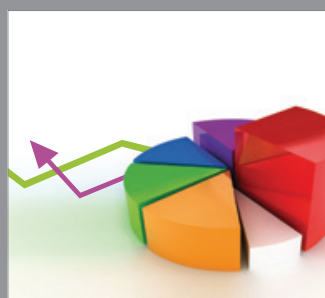

ournal of

Probability and Statistics

Promensencen
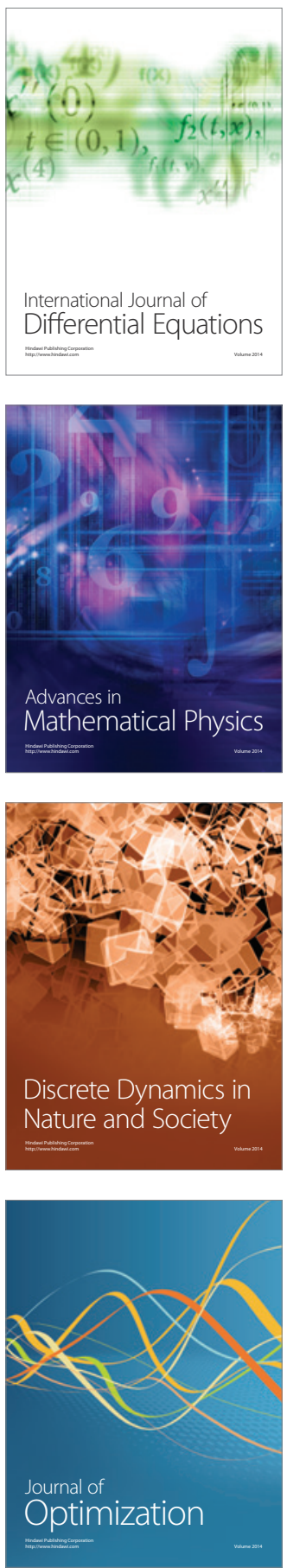